\title{
A practical scheme to introduce explicit tidal forcing into an OGCM
}

\author{
K. Sakamoto, H. Tsujino, H. Nakano, M. Hirabara, and G. Yamanaka \\ Meteorological Research Institute, Nagamine, Tsukuba, Ibaraki 305-0052, Japan \\ Correspondence to: K. Sakamoto (ksakamot@mri-jma.go.jp)
}

Received: 14 February 2013 - Published in Ocean Sci. Discuss.: 7 March 2013

Revised: 4 October 2013 - Accepted: 22 October 2013 - Published: 13 December 2013

\begin{abstract}
A practical scheme is proposed to explicitly introduce tides into ocean general circulation models (OGCM). In this scheme, barotropic linear response to the tidal forcing is calculated by the time differential equations modified for ocean tides, instead of the original barotropic equations of an OGCM. This allows for the usage of various parameterizations specified for tides, such as the self-attraction/loading (SAL) effect and energy dissipation due to internal tides, without unintentional violation of the original dynamical balances in an OGCM. Meanwhile, secondary nonlinear effects of tides, e.g., excitation of internal tides and advection by tidal currents, are fully represented within the framework of the original OGCM equations. That is, this scheme drives the OGCM by the barotropic linear tidal currents which are predicted progressively by a tuned tide model, instead of the equilibrium tide potential, without large additional numerical costs. We incorporated this scheme into Meteorological Research Institute Community Ocean Model and executed test experiments with a low-resolution global model. The results showed that the model can simulate both the non-tidal circulations and the tidal motion simultaneously. Owing to the usage of tidal parameterizations such as a SAL term, a rootmean-squared error in the tidal heights is found to be as small as $10.0 \mathrm{~cm}$, which is comparable to that of elaborately tuned tide models. In addition, analysis of the speed and energy of the barotropic tidal currents is found to be consistent with that of past tide studies. The model also showed active excitement of internal tides and tidal mixing. In the future, the impacts of internal tides and tidal mixing should be examined using a model with a finer resolution, since explicit and precise introduction of tides into an OGCM is a significant step toward the improvement of ocean models.
\end{abstract}

\section{Introduction}

Recent advances in theories of ocean general circulations and observations of deep seas have revealed that tides play a significant role in open oceans as well as in coastal areas. As a representative study, Munk and Wunsch (1998) suggested that vertical mixing in deep seas due to breaking of internal tides is an important process in the global thermohaline circulations. This hypothesis is supported by the fact that a large part of the tidal energy is dissipated in deep seas (Egbert and Ray, 2001; St. Laurent and Garrett, 2002; Niwa and Hibiya, 2011). In addition, various studies reported that local strong tidal mixing affects the ocean circulations on a basin scale. For example, tidal mixing near the Kuril Islands plays an important role in the formation process of the water mass called the North Pacific Intermediate Water (Nakamura and Awaji, 2004; Osafune and Yasuda, 2006). Tidal mixing in the Arctic Shelf seas modifies the salinity budget through interaction with sea ice, and, as a result, the deep thermohaline circulation in the North Atlantic (Postlethwaite et al., 2011). In a similar fashion, tidal mixing over the Antarctic shelves affects the formation process of Antarctic Bottom Water (Robertson, 2001a, b; Pereira et al., 2002). These studies suggest an influence of tides on the general circulation.

However, only recently have tides begun to be sufficiently taken into account in ocean general circulation models (OGCMs). One reason is that most OGCMs could not represent tides since they adopted the rigid-lid condition to preclude the surface gravity waves due to the CourantFriedrichs-Lewy condition. Another reason is that tidal motions with a time scale of half a day or one day were intentionally omitted to focus on variations with longer time scales in geostrophic currents. Recently, due to advances in the studies of tides, the effects of tides have begun to be considered in OGCMs. 


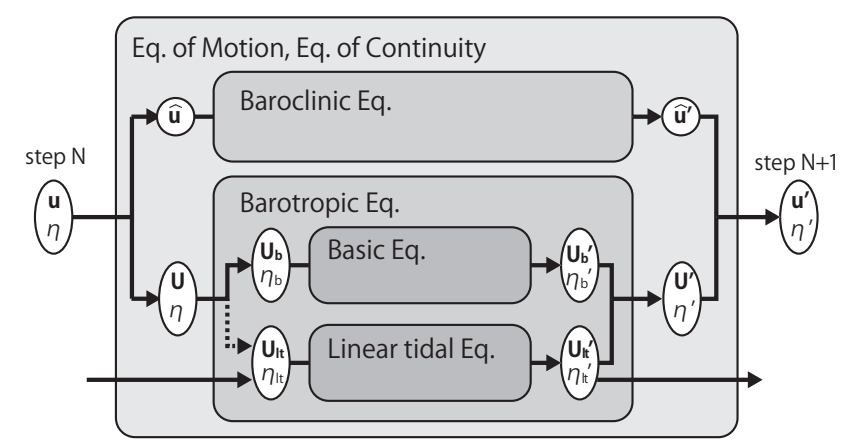

Fig. 1. A schematic view of the calculation procedure of the tide scheme. From $\boldsymbol{u}$ and $\eta$ at the time step $N, \boldsymbol{u}^{\prime}$ and $\eta^{\prime}$ at the next step $N+1$ are calculated following the equations of motion and continuity. In the calculation process, the mode splitting technique splits the variables into the baroclinic constituent, $\hat{\boldsymbol{u}}$, and the barotropic one, $\boldsymbol{U}$ and $\eta$, and then the tide scheme splits the latter into the basic component, $\boldsymbol{U}_{b}$ and $\eta_{b}$, and the linear tidal component, $\boldsymbol{U}_{l t}$ and $\eta_{l t}$. Each individual component calculates time evolution $\left(\hat{\boldsymbol{u}}^{\prime}, \boldsymbol{U}_{b}^{\prime}, \eta_{b}^{\prime}, \boldsymbol{U}_{l t}^{\prime}\right.$ and $\left.\eta_{l t}^{\prime}\right)$, and subsequently all of the time evolution values are summed to obtain $\boldsymbol{u}^{\prime}$ and $\eta^{\prime}$. The dashed and solid arrows which point to $\boldsymbol{U}_{l t}$ and $\eta_{l t}$ indicate that their time evolution values are given almost independently (see the main text).

The methodology of incorporating tides into OGCMs is classified into two types, i.e., an implicit one and an explicit one. Models with the implicit type use indirect parameterizations about tidal effects rather than simulating tides themselves, in order to avoid drastically changing the OGCM framework. A typical parameterization adopted by various recent OGCMs is a mixing enhancement in deep seas and coastal areas (e.g., St. Laurent and Garrett, 2002). Lee et al. (2006) reported that this kind of parameterization contributes to good representation of the salinity distribution in the North Atlantic. As another indirect parameterization, Bessiéres et al. (2008) proposed an implicit parameterization for the tidal residual currents.

The explicit type introduces the tidal dynamics into freesurface OGCMs directly; i.e., through introduction of tidal forcing in the momentum equations. Though this type needs large computer resources and modification of a part of the OGCM framework, some achievements have been already reported. For example, Schiller and Fiedler (2007) improved model representation of water transport and mixing in the Indonesian Through Flow region and Australian shelves. Müller et al. (2010) reported improvement in modeling of the pathway of the North Atlantic Current and watermodification processes in the North Atlantic. In addition, Arbic et al. (2010) discussed a possibility that explicit incorporation of tides into an eddy-resolving OGCM may lead to drastic improvement in the representation of various ocean processes - such as interaction between meso-scale eddies and tides, form drag on the sea mounts, and the excitement and propagation of internal tides - in realistic three- dimensional stratification. Development of an OGCM, which simultaneously simulates the time evolution of the tidal field and the non-tidal field (called the basic field hereafter), is now a frontier in ocean modeling.

By contrast, the modeling of tides themselves has been developed virtually independently of OGCMs, based on barotropic ocean models. Many modeling studies have shown that dynamics particular to tides need to be introduced into the model equations for accurate representation of tides (e.g., Matsumoto et al., 2000). A typical example is the selfattraction/loading (SAL) effect. This represents modification of the gravity field and elastic deformation of the bottom ground induced by movement of ocean water (Schwiderski, 1980). Due to the SAL effect, the pressure gradient term accompanied by the tidal height gradient, $-\mathrm{g} \nabla \eta$, is modified in the equation of barotropic motion as follows:

$-g \nabla\left(\eta-\eta_{\mathrm{SAL}}\right)$

where $\eta$ is the sea surface height (SSH) anomaly (m) and $g$ is the gravitational acceleration $\left(\mathrm{m} \mathrm{s}^{-2}\right.$ ) (See Table 1 for variables and constants in this paper). In order to represent the gravity change of the self-attraction and the loading effect - which is that the sea surface elevation induced by convergence of barotropic velocities is partially canceled by depression of the bottom ground due to water weight - the elevation is subtracted by $\eta_{\mathrm{SAL}}$ in the calculation of the pressure gradient. Though various evaluations of $\eta_{\text {SAL }}$ have been proposed, a linear response is used as a first-order approximation,

$\eta_{\mathrm{SAL}}=(1-\alpha) \eta$

where $\alpha$ is a constant between 0.88 and 0.95 (Matsumoto et al., 2000). Under this approximation of the SAL term, which has been traditionally referred to as the "scalar approximation" (Hendershott, 1972), the pressure gradient term $-g \nabla \eta$ is modified to $-g \alpha \nabla \eta$.

Another issue of tide modeling is energy dissipation of tides, such as energy transfer to internal tides and form drag by bottom topography on tidal currents. In general, as model resolution becomes finer, a model can represent more kinds of dissipation processes without parameterization. However, considering that internal tide processes cannot be reproduced sufficiently even in a model with a horizontal resolution of $10 \mathrm{~km}$, which is considerably fine at present (e.g. Niwa and Hibiya, 2011), a parameterization specialized for dissipation is still necessary to represent tides with good accuracy (Jayne and St. Laurent, 2001; Arbic et al., 2004). Furthermore, various parameterizations unusual for OGCMs have been proposed for tidal modeling, such as body tides, which are included here, and atmospheric tides. See Chapter 6 of Kantha and Clayson (2000) for a detailed description.

The knowledge obtained by tidal modeling studies should be exploited in order to introduce tides into OGCMs with high accuracy. However, this is a difficult task, since dynamical balances in the basic field of OGCMs are violated if the 
Table 1. Mathematical variables and constants in the main text and Appendix.

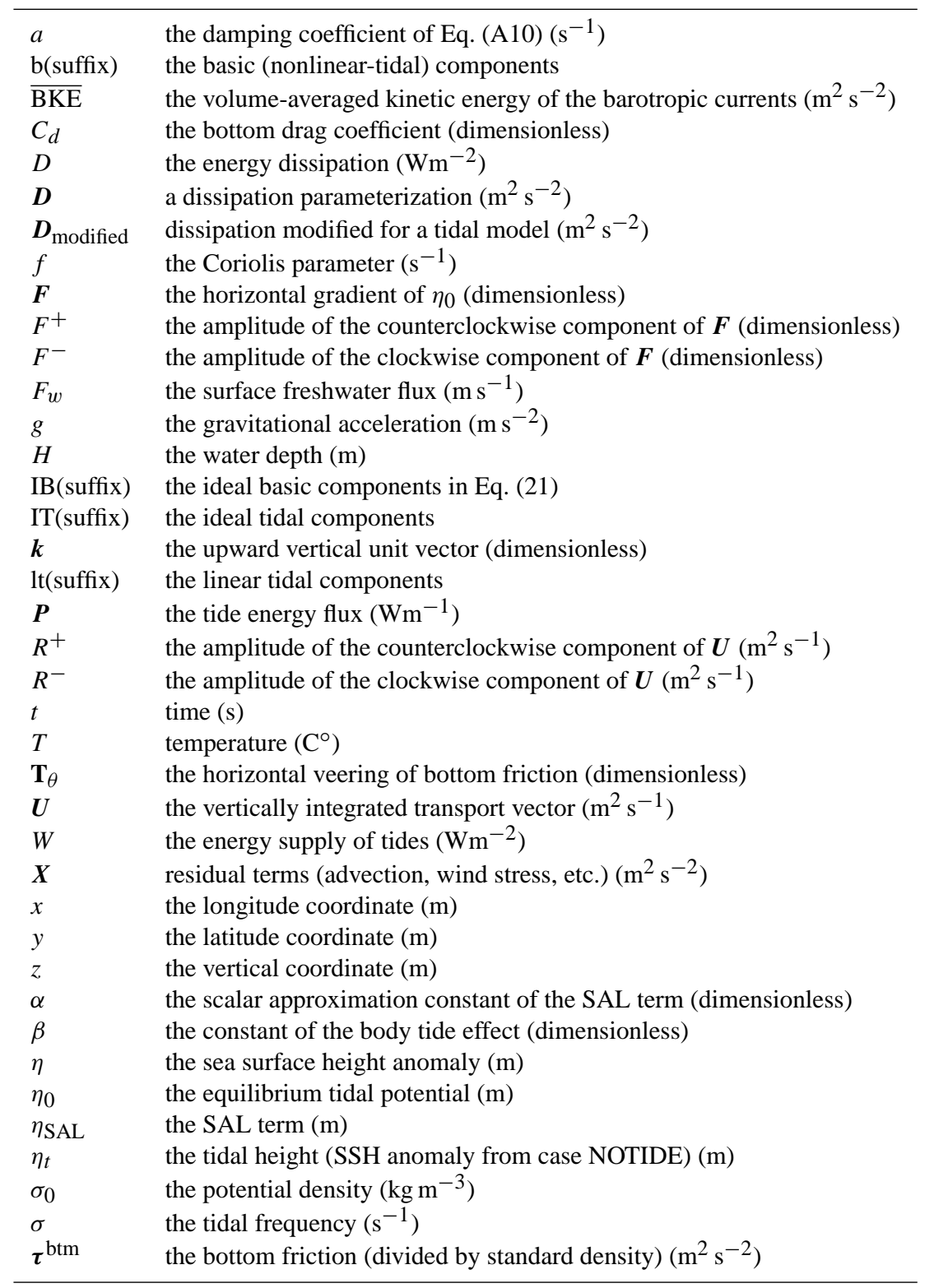

proposed terms for tidal modeling are incorporated into the OGCM equations directly (Arbic et al., 2010). For example, the SAL term of Eq. (1) changes the geostrophic relationship between sea surface gradient and currents. Parameterizations for dissipation of tidal currents are not suited for the geostrophic currents in OGCMs either, since their time scales of change are so different from each other that their dissipation mechanisms are not the same. Therefore, we cannot simply replace the governing equations of OGCMs by those of tidal modeling. The two sets of the governing equations should be harmonized by some means in order to introduce tides into an OGCM.
This problem has not yet been solved. In most of the model studies that explicitly introduce tides into OGCMs, the equilibrium tidal potential is given directly through the freesurface barotropic equation of motion, and the tidal and basic fields are calculated without separation (Thomas et al., 2001; Schiller, 2004; Schiller and Fiedler, 2007; Müller et al., 2010). In these studies, the problem due to the differences between the tidal and basic (geostrophic and eddying) characteristics is not investigated sufficiently. To the best of our knowledge, Arbic et al. (2010), who introduced tides into an eddy-resolving global OGCM, examined this problem most carefully. Though they calculated time evolution of the tidal and basic fields without separation as in other studies, they 
elaborated a method to prevent the parameterizations specialized for tides from affecting the basic fields. Specifically, they defined the tidal currents as velocity deviations from a 25-hour running mean (which is calculated progressively in the model), and restricted the tidal dissipation parameterization to work on the tidal currents only. In addition, they defined the tidal height as the SSH deviation from the dynamical height (which is calculated every time step), and evaluated the SAL term so that the SAL would not contaminate the basic field (e.g., geostrophic currents). However, their method is expected to expend a substantial amount of numerical resources. Furthermore, it seems questionable that all of the SSH deviation is treated as tidal height.

As a solution to the aforementioned problem, we propose a new practical scheme to incorporate tides explicitly into OGCMs. This scheme is based on the recognition that the governing equations are different between the tidal and basic fields, and calculates their time evolutions separately. This approach is in contrast to traditional typical schemes such as that by Schiller (2004), in which the tidal and basic fields are given by the same governing equations.

First this paper will explain our scheme in detail. Next, model representations of tides by this scheme will be shown based on some test experiments of a global OGCM. Finally, tidal effects on the basic fields in the OGCM will be presented briefly.

\section{Scheme and model}

\subsection{Conventional scheme}

Before presenting the new tide scheme, we will discuss the scheme of Schiller (2004) as a representative example of the traditional schemes in which the tidal forcing is incorporated directly into the governing equations. First, the original standard expressions of the barotropic equations of motion and continuity are

$$
\begin{aligned}
& \frac{\partial \boldsymbol{U}}{\partial t}+f \boldsymbol{k} \times \boldsymbol{U}=-g(\eta+H) \nabla \eta+\boldsymbol{D}+\boldsymbol{\tau}^{\mathrm{btm}}+\boldsymbol{X} \\
& \frac{\partial \eta}{\partial t}+\nabla \cdot \boldsymbol{U}=F_{\mathrm{w}},
\end{aligned}
$$

where $t$ is the time (s), $\boldsymbol{U}$ the vertically integrated transport vector $\left(\mathrm{m}^{2} \mathrm{~s}^{-1}\right), f$ the Coriolis parameter $\left(\mathrm{s}^{-1}\right), \boldsymbol{k}$ the upward vertical unit vector (dimensionless), $H$ the water depth (m), $\boldsymbol{D}$ a dissipation parameterization $\left(\mathrm{m}^{2} \mathrm{~s}^{-2}\right), \boldsymbol{\tau}^{\mathrm{btm}}$ the bottom friction (already divided by standard density) $\left(\mathrm{m}^{2} \mathrm{~s}^{-2}\right)$, $\boldsymbol{X}$ the other residual terms including the vertically integrated advection and the wind stress $\left(\mathrm{m}^{2} \mathrm{~s}^{-2}\right)$, and $F_{w}$ the surface freshwater flux $\left(\mathrm{m} \mathrm{s}^{-1}\right)$. Introduction of tides means that the equilibrium tidal potential $\eta_{0}$ and the SAL term $\eta_{\mathrm{SAL}}$ are added, and $\boldsymbol{D}$ is changed to the dissipation parameterization specialized for tides, which is indicated by $\boldsymbol{D}_{\text {modified, as }}$

$$
\begin{aligned}
\frac{\partial \boldsymbol{U}}{\partial t} & +f \boldsymbol{k} \times \boldsymbol{U}=-g(\eta+H) \nabla\left(\eta-\beta \eta_{0}-\eta_{\mathrm{SAL}}\right) \\
& +\boldsymbol{D}_{\text {modified }}+\boldsymbol{\tau}^{\mathrm{btm}}+\boldsymbol{X},
\end{aligned}
$$

where $\beta$ represents the body tide effect (Schwiderski, 1980). If the scalar approximation for the SAL term, Eq. (2), is adopted, Eq. (6) becomes

$$
\begin{aligned}
\frac{\partial \boldsymbol{U}}{\partial t} & +f \boldsymbol{k} \times \boldsymbol{U}=-g(\eta+H) \nabla\left(\alpha \eta-\beta \eta_{0}\right) \\
& +\boldsymbol{D}_{\text {modified }}+\boldsymbol{\tau}^{\mathrm{btm}}+\boldsymbol{X} .
\end{aligned}
$$

This is equivalent to a standard barotropic tidal model, e.g., the continuous ocean tide equations of Schwiderski (1980). In this traditional scheme, the time evolutions of $\boldsymbol{U}$ and $\eta$ under the tidal forcing are obtained by solving Eqs. (4) and (6) (or (7)) under $\eta_{0}(x, y, t)$, which is analytically calculated.

This scheme works well for modeling of tides without basic circulation. However, in modeling of the tidal and basic fields simultaneously, it induces the problem that the terms specialized for tides affect the basic fields unintentionally. In fact, Eq. (7) clearly shows that the SAL term changes the relationship between the sea surface gradient and the barotropic currents. The dissipation, $\boldsymbol{D}_{\text {modified, }}$ also changes the basic currents. Introduction of parameterizations specified for tides results in violation of the dynamical balances in the basic fields.

\subsection{New scheme}

The violation of the dynamical balance in the basic fields arises from the fact that the barotropic equation of motion for tides, Eq. (6), is different from the OGCM standard equation for the basic fields, Eq. (3). Therefore, our new tide scheme calculates the tidal and basic fields by two different equations as explained below. The objective of our new scheme is to simultaneously achieve both accurate modeling of the tides and maintenance of the dynamical balances in the original OGCM.

The basis of the scheme is decomposition of the variables, $\boldsymbol{U}, \eta, \boldsymbol{D}$ and $\boldsymbol{\tau}^{\mathrm{btm}}$ in the barotropic equations into the linear tidal component and the basic component,

$$
\begin{gathered}
\boldsymbol{U}=\boldsymbol{U}_{b}+\boldsymbol{U}_{l t} \\
\eta=\eta_{b}+\eta_{l t} \\
\boldsymbol{D}=\boldsymbol{D}_{b}+\boldsymbol{D}_{l t} \\
\boldsymbol{\tau}^{\mathrm{btm}}=\boldsymbol{\tau}_{b}^{\mathrm{btm}}+\boldsymbol{\tau}_{l t}^{\mathrm{btm}}
\end{gathered}
$$

The linear tidal component, indicated by the subscript " $l t$ ", corresponds to the primary response of the barotropic ocean to the equilibrium tide potential. The basic component with the subscript, " $b$ ", corresponds to the other barotropic and baroclinic motions, including all of the dynamical processes in the original OGCM and the secondary effects of tides (e.g., internal tides, tidal advection, bottom shear of tidal currents, 
and so on). There are some difficulties posed by the decomposition of the variables, especially $\tau^{\mathrm{btm}}$, and this decomposition is discussed in more detail later.

Each of the two components is calculated using its own governing equation. The linear tidal component is governed by the equations for tidal modeling; i.e., a modified Eq. (6) and the continuity equation:

$$
\begin{aligned}
& \frac{\partial \boldsymbol{U}_{l t}}{\partial t}+f \boldsymbol{k} \times \boldsymbol{U}_{l t}=-g(\eta+H) \nabla\left(\eta_{l t}-\beta \eta_{0}-\eta_{\mathrm{SAL}}\right) \\
&+\boldsymbol{D}_{l t}+\boldsymbol{\tau}_{l t}^{\mathrm{btm}} \\
& \frac{\partial \eta_{l t}}{\partial t}+\nabla \cdot \boldsymbol{U}_{l t}=0 .
\end{aligned}
$$

The basic component is governed by the standard OGCM equations, i.e., Eqs. (3) and (4).

$$
\begin{aligned}
& \frac{\partial \boldsymbol{U}_{b}}{\partial t}+f \boldsymbol{k} \times \boldsymbol{U}_{b}=-g(\eta+H) \nabla \eta_{b}+\boldsymbol{D}_{b}+\boldsymbol{\tau}_{b}^{\mathrm{btm}}+\boldsymbol{X} \\
& \frac{\partial \eta_{b}}{\partial t}+\nabla \cdot \boldsymbol{U}_{b}=F_{w}
\end{aligned}
$$

The numerical procedure to predict the two components by the equations above is schematically illustrated by Fig. 1 . Generally in a free-surface OGCM, if one starts with velocity $\boldsymbol{u}$ and SSH $\eta$ at the time step $N$, the velocity and SSH at the next step, $N+1$, are calculated using the equations of motion and continuity, and usually the barotropic and baroclinic constituents are predicted differently in the calculation (so called mode splitting, indicated by $\hat{\boldsymbol{u}}, \boldsymbol{U}$ and $\eta$ in Fig. 1). The key to our new scheme is to further split the barotropic constituent into the basic and linear tidal components $\left(\boldsymbol{U}_{b}, \eta_{b}, \boldsymbol{U}_{l t}\right.$ and $\eta_{l t}$ ), and to calculate the time evolution of $\boldsymbol{U}_{l t}$ and $\eta_{l t}$ separately from $\boldsymbol{U}_{b}$ and $\eta_{b}$. The solid arrow connected to $\boldsymbol{U}_{l t}$ and $\eta_{l t}$ in Fig. 1 indicates this independent calculation, whereas the dashed arrow means that $\boldsymbol{U}_{b}$ and $\eta_{b}$ are given by subtraction, i.e., $\boldsymbol{U}-\boldsymbol{U}_{l t}$ and $\eta-\eta_{l t}$, respectively. That is, the three sets of the governing equations are calculated at each time step, and the three-dimensional velocity field at the next step is determined by their summation.

The linear terms in the barotropic equations, such as the Coriolis force and the Laplacian horizontal viscosity, can be split into the basic and linear tidal components naturally, while the nonlinear terms need to be treated more carefully. In our scheme, all of the advection terms are incorporated into the basic equations ( $\boldsymbol{X}$ in Eq. (13)), and the linear tidal equations have no advection. Specifically, the tracer and momentum advections are calculated using the threedimensional velocity field, given by the summation of all of their components ( $\boldsymbol{u}$ in Fig. 1), and these sums are added to the basic equations. This is based on the assumption of the following scheme: the linear tidal component represents only the linear primary response to the tidal forcing, and the other secondary effects, such as tidal advection and internal tides, are represented by the basic component. Modification of the tides due to interaction between tidal currents and basic fields is also represented by the basic equations as secondary oscillations with tidal frequencies (see Appendix for detail).

The wind stress (included in $\boldsymbol{X}$ ) and the freshwater flux $F_{w}$ are also left in the original OGCM equations, Eqs. (13) and (14). This is because the wind-induced circulations and the thermohaline circulations induced by these terms should be represented in the OGCM framework. If these terms were moved to the linear tidal equations, the dynamical balance would be violated due to tidal parameterizations such as the SAL term. In addition, as far as we know, it has not been reported that these terms change the primary response of the ocean to the tidal forcing. Nevertheless, various processes of secondary interaction between tides and basic fields have been reported, e.g., influence of tidal mixing on a thermohaline circulation (Lee et al., 2006) and modification of internal tides induced by winds (Xing and Davies, 1997). These kinds of interaction processes are intended to be represented under the OGCM equations.

Decomposition of the bottom friction $\boldsymbol{\tau}^{\mathrm{btm}}$ should be carefully treated, since it is nonlinear, when expressed by a quadratic form as (Taylor, 1920; Weatherly et al., 1980)

$\boldsymbol{\tau}^{\mathrm{btm}}=-C_{D}\left|\frac{\boldsymbol{U}}{H+\eta}\right| \mathbf{T}_{\theta} \frac{\boldsymbol{U}}{H+\eta}$.

The constant $C_{D}$ indicates a drag coefficient and the $\mathbf{T}_{\theta}$ indicates a matrix representing horizontal veering,

$\mathbf{T}_{\theta}=\left(\begin{array}{cc}\cos \theta & -\sin \theta \\ \sin \theta & \cos \theta\end{array}\right)$,

where $\theta$ is the veer angle. (The unit $\boldsymbol{\tau}^{\mathrm{btm}}$ is given in $\mathrm{m}^{2} \mathrm{~s}^{-2}$, and $C_{D}$ and $\mathbf{T}_{\theta}$ are dimensionless.) There are various ways to split Eq. (15) into the term for the basic barotropic equation and that for the linear tidal equation. For simplicity of the equations, we decided that a sum of the two components would be used for $|\boldsymbol{U} /(H+\eta)|$ (the coefficient part), but each component for $\boldsymbol{U} /(H+\eta)$ (the vector part) would be given by

$$
\begin{aligned}
\boldsymbol{\tau}_{b}^{\mathrm{btm}} & =-C_{D}\left|\frac{\boldsymbol{U}_{b}+\boldsymbol{U}_{l t}}{H+\eta}\right| \mathbf{T}_{\theta} \frac{\boldsymbol{U}_{b}}{H+\eta} \\
\boldsymbol{\tau}_{l t}^{\mathrm{btm}} & =-C_{D}\left|\frac{\boldsymbol{U}_{b}+\boldsymbol{U}_{l t}}{H+\eta}\right| \mathbf{T}_{\theta} \frac{\boldsymbol{U}_{l t}}{H+\eta} .
\end{aligned}
$$

It should be noted that $\boldsymbol{\tau}_{l t}^{\mathrm{btm}}$ depends on $\boldsymbol{U}_{b}$ through the coefficient part. In open oceans, it is assumed that this dependency is not important, since both of the barotropic velocities, $\boldsymbol{U}_{b} /(H+\eta)$, and $\boldsymbol{\tau}_{l t}^{\mathrm{btm}}$ itself are generally small. In coastal regions, however, these variables become large, and consequentially the reproducibility of the tides may be affected to some extent. (We investigated the affect using our low-resolution model by replacing the coefficient part of Eq. (18) by $\left|\boldsymbol{U}_{l t} /(H+\eta)\right|$. In a one-year experiment, change of the tidal heights is found to be $1 \%$ at most.) Through the 
decomposition, we make the linear tidal equations, Eqs. (11) and (12), as simple as possible, in order to represent only the primary barotropic response to the tidal forcing.

Using our new scheme, we can avoid the violation of the dynamical balance in the basic field. To show this achievement more clearly, we assume the SAL term has a linear form as $\eta_{\mathrm{SAL}} \sim(1-\alpha) \eta_{l t}$, and sum Eqs. (13) and (12) to find that

$$
\begin{aligned}
\frac{\partial \boldsymbol{U}}{\partial t} & +f \boldsymbol{k} \times \boldsymbol{U}=-g(\eta+H) \nabla\left(\eta_{b}+\alpha \eta_{l t}-\beta \eta_{0}\right) \\
& +\boldsymbol{D}_{b}+\boldsymbol{D}_{l t}+\boldsymbol{\tau}_{b}^{\mathrm{btm}}+\boldsymbol{\tau}_{l t}^{\mathrm{btm}}+\boldsymbol{X} .
\end{aligned}
$$

This equation for motion is clearly different from the conventional scheme, Eq. (7). The SAL effect $(\alpha)$ works on $\eta_{l t}$ only, the body tide effect works on the equilibrium tide only, and the expressions for dissipation and bottom friction for tides $\left(\boldsymbol{D}_{l t}\right.$ and $\left.\boldsymbol{\tau}_{l t}^{\mathrm{btm}}\right)$ are different from the basic field $\left(\boldsymbol{D}_{b}\right.$ and $\boldsymbol{\tau}_{b}^{\mathrm{btm}}$ ). As a result, when tides are omitted (i.e., $\eta_{0} \equiv 0$ ), $\boldsymbol{U}_{l t}, \eta_{l t}, \boldsymbol{D}_{l t}$ and $\boldsymbol{\tau}_{l t}^{\mathrm{btm}}$ are permanently zero in Eqs. (11), (12) and (18), so that Eq. (19) becomes identical to the original barotropic equation, Eq. (3). Thus, the introduction of our tide scheme, in contrast to conventional tide schemes, does not modify the basic equations.

From the point of view of tidal modeling, our scheme enables us to tune the parameterizations of tides independently of the dynamical balance in the basic field. The value of $\alpha$, the formulation of $\boldsymbol{\tau}_{l t}^{\mathrm{btm}}$ and the parameterization of $\boldsymbol{D}_{l t}$ can be selected independently.

To clarify the base of the new scheme, the meaning of "the linear tidal component" is noted again here. Strictly speaking, a part of the basic component of $\eta$ is used in Eqs. (11) and (12) so the linear tidal component is not strictly independent of the basic component. However, the linear tidal component is treated separately from the basic component. In other words, the scheme calculates the linear tidal currents under the equilibrium tidal potential progressively, and uses it as a model forcing, instead of introducing the potential to the model directly. That is, the linear tidal component can be referred to as an external forcing for the model, rather than the tidal field reproduced in the model. To see the tidal field precisely, secondary oscillations with tidal frequencies in the basic field need to be taken into account, as explained in the Appendix.

In closing this subsection, the practical approximation used by our new scheme is compared with the scheme of Arbic et al. (2010) in detail. The first principle of our new scheme is that the different sets of the governing equations should be applied to the tidal component and the nontidal component separately, in order to introduce tides into OGCMs realistically. To do so, we have to carry out decomposition of the two components in an OGCM. In an ideal scheme, the tidal component would represent all of the barotropic motions which oscillate with tidal frequencies and have the spatial structures corresponding to the tidal forcing, while the non-tidal component would represent all of the other motions. Hereafter, we call these components "the ideal tidal component" and "the ideal basic component", respectively. Under such an ideal decomposition, the barotropic equation comparable to Eq. (19) becomes

$$
\begin{gathered}
\frac{\partial \boldsymbol{U}}{\partial t}+f \boldsymbol{k} \times \boldsymbol{U}=-g(\eta+H) \nabla\left(\eta_{\mathrm{IB}}+\alpha \eta_{\mathrm{IT}}-\beta \eta_{0}\right) \\
+\boldsymbol{D}_{\mathrm{IB}}+\boldsymbol{D}_{\mathrm{IT}}+\boldsymbol{\tau}_{\mathrm{IB}}^{\mathrm{btm}}+\boldsymbol{\tau}_{\mathrm{IT}}^{\mathrm{btm}}+\boldsymbol{X}_{\mathrm{IB}}+\boldsymbol{X}_{\mathrm{IT}},
\end{gathered}
$$

where the variables with the "IB" and "IT" subscripts indicate the ideal basic component and the ideal tidal component, respectively. However, it is virtually impossible to extract the ideal tidal component (i.e., all of the motions with tidal frequencies and with spatial patterns corresponding to the tidal forcing), from the changing model results. A certain approximation is necessary for the decomposition.

In the scheme of Arbic et al. (2010), the decomposition is executed in a straightforward manner. Arbic et al. (2010) approximated terms in Eq. (20) as follows

$$
\left\{\begin{array}{l}
\eta_{\mathrm{IB}}=\eta_{\text {dynamic-height }} \\
\eta_{\mathrm{IT}}=\eta-\eta_{\text {dynamic }} \text {-height } \\
\boldsymbol{D}_{\mathrm{IB}}=\boldsymbol{D}_{25 \text { h-mean-current }} \\
\boldsymbol{D}_{\mathrm{IT}}=\boldsymbol{D}_{\text {anomaly-current }}
\end{array}\right.
$$

where $\eta_{\text {dynamic-height }}$ indicates the dynamic height anomaly,

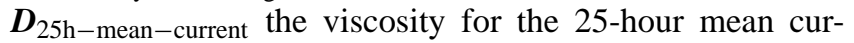
rents, and $\boldsymbol{D}_{\text {anomaly-current }}$ the viscosity for the anomalous currents. It is guessed that the bottom friction, $\boldsymbol{\tau}^{\mathrm{btm}}$, and the other (nonlinear) term $\boldsymbol{X}$ are formulated in the same manner for the two components. In an OGCM with the Arbic et al. (2010) tide scheme, Eq. (20) is calculated under these approximations.

Meanwhile, our new scheme approximates the terms of Eq. (20) as follows:

$$
\left\{\begin{array}{l}
\eta_{\mathrm{IB}}=\eta_{b}=\eta-\eta_{l t} \\
\eta_{\mathrm{IT}}=\eta_{l t} \\
\boldsymbol{D}_{\mathrm{IB}}=\boldsymbol{D}_{b} \quad\left(\text { for } \boldsymbol{U}_{b}=\boldsymbol{U}-\boldsymbol{U}_{l t}\right) \\
\boldsymbol{D}_{\mathrm{IT}}=\boldsymbol{D}_{l t} \quad\left(\text { for } \boldsymbol{U}_{l t}\right) \\
\boldsymbol{\tau}_{\mathrm{IB}}^{\mathrm{btm}}=\boldsymbol{\tau}_{b}^{\mathrm{btm}} \quad\left(\text { for } \boldsymbol{U}_{b}\right) \\
\boldsymbol{\tau}_{\mathrm{IT}}^{\mathrm{btm}}=\boldsymbol{\tau}_{l t}^{\mathrm{btm}} \quad\left(\text { for } \boldsymbol{U}_{l t}\right) \\
\boldsymbol{X}_{\mathrm{IB}}=\boldsymbol{X} \quad\left(\text { for } \boldsymbol{U}_{b}+\boldsymbol{U}_{l t}\right) \\
\boldsymbol{X}_{\mathrm{IT}}=0
\end{array}\right.
$$

by introducing the barotropic linear tidal component, made up of $\eta_{l t}$ and $\boldsymbol{U}_{l t}$, and the equations Eqs. (11) and (12) to calculate both elements' time evolution. That is, the practical approximation of our new scheme is to use the solution of a linear tidal model in the decomposition. It is because of this approximation that tidal fields can be reproduced with less numerical resources and be accurate enough to represent tidal effects in an OGCM, as shown in Sect. 3. However, it may be difficult to reproduce coastal-area tidal fields in detail; since nonlinear effects become important in coastal areas, the linear tidal component becomes less representative.

Though relatively small, some numerical resources are expended by the scheme. Since one more barotropic equation 
needs to be calculated as shown in Fig. 1, the numerical cost of the barotropic calculation doubles. For our test experiment, the computational time increased by $4 \%$ overall.

\subsection{Model}

Test experiments of our tide scheme were executed using the Meteorological Research Institute Community Ocean Model (MRI.COM) (Tsujino et al., 2010, 2011). The MRI.COM is a hybrid $\mathrm{z}-\sigma$ coordinate free-surface multilevel model which solves the primitive equations under the hydrostatic and Boussinesq approximations, and adopts a barotropicbaroclinic mode-splitting technique. The model domain is global (with so-called tripolar grid coordinates (Murray, 1996)). The horizontal resolution is $1^{\circ}$ in the zonal direction and $1 / 2^{\circ}$ in the meridional direction, except for the Arctic region. The model has 50 levels in the vertical direction, with layer thickness increasing from $4 \mathrm{~m}$ at the surface to $600 \mathrm{~m}$ at $6300 \mathrm{~m}$ depth. The model settings are like those of recent global OGCMs except for the tide scheme as follows (see Tsujino et al. (2011) for details). The model uses isopycnal diffusion (Gent and Mcwilliams, 1990), the second-order moment tracer advection of Prather (1986), harmonic friction with a Smagorinsky-like viscosity (Griffies and Hallberg, 2000), a bottom boundary layer scheme (Nakano and Suginohara, 2002), and the generic length scale vertical mixing scheme (Umlauf and Burchard, 2003). A sea ice model is also incorporated. Its thermodynamic part is based on Mellor and Kantha (1989), and the other dynamic processes, such as categorization by thickness, ridging and rheology, are based on the Los Alamos sea ice model (Hunke and Dukowicz, 1997, 2002). The bottom friction for the basic field, $\boldsymbol{\tau}_{b}^{\mathrm{btm}}$, is represented by the quadratic friction Eq. (15) with $C_{D}=0.00125$ and $\theta=10^{\circ}$ (Weatherly et al., 1980).

Configurations of the tide scheme are rather simple in order to verify its basic features. The SAL term is approximated by the linear form $\eta_{\mathrm{SAL}}=(1-\alpha) \eta_{l t}$ with $\alpha=0.88$, and the constant $\beta$ is approximated as 0.7 . (Strictly speaking, $\beta$ should depend on the Love numbers (Chapter 6.3 of Kantha and Clayson, 2000).) A simple harmonic horizontal viscosity is used for the diffusivity term, $\boldsymbol{D}_{l t}$, with a coefficient of $6 \times 10^{4} \mathrm{~m}^{2} \mathrm{~s}^{-1}$ (although more sophisticated parameterizations have been proposed, such as a formulation dependent on the mixing length (Schwiderski, 1980)). The no-slip condition is imposed at the lateral boundary of the bottom topography, so that the horizontal viscosity works there. The bottom friction for the linear tidal component $\boldsymbol{\tau}_{l t}^{\mathrm{btm}}$ is the quadratic friction with the conventional parameters $C_{D}=0.0025$ and $\theta=0^{\circ}$ (Schwiderski, 1980). Thus, $\boldsymbol{\tau}_{l t}^{\mathrm{btm}}$ uses a different parameterization from $\boldsymbol{\tau}_{b}^{\mathrm{btm}}$, since the Weatherly et al. (1980) scheme used for $\boldsymbol{\tau}_{b}^{\mathrm{btm}}$ is designed for the turbulent Ekman layer and unsuitable for the tidal currents (Sakamoto and Akitomo, 2008, 2009). The main eight tidal constituents (K1, O1, P1, Q1, M2, S2, N2 and K2) are
Table 2. Experimental cases simulated using our new scheme with MRI.COM.

\begin{tabular}{ll}
\hline Abbreviation & settings \\
NOTIDE & without tides \\
TIDE & 8 tidal constituents \\
TIDEa1 & 8 tidal constituents, $\alpha=1$ \\
M2 & M2 \\
K1 & K1 \\
M2v2 & M2, horizontal viscosity $=2 \times 10^{4} \mathrm{~m}^{2} \mathrm{~s}^{-1}$ \\
M2v10 & M2, horizontal viscosity $=1 \times 10^{5} \mathrm{~m}^{2} \mathrm{~s}^{-1}$ \\
\hline
\end{tabular}

used for the equilibrium tide potential, and their amplitudes and phases are given after Table 1 of Schwiderski (1980). ${ }^{1}$

\subsection{Experimental cases}

The test experiments were executed under the following boundary and initial conditions. The atmospheric forcings such as wind stress, latent and sensible heat fluxes, evaporation, and precipitation - were calculated using the interannual data set of the Coordinated Ocean-ice Reference Experiments (Griffies et al., 2009) and the bulk formulas of Large and Yeager (2004). For spin up, we ran the model without tides over 1000 years under repeated atmospheric forcings with climatological temperature and salinity in order to reach a quasi-steady realistic situation from a state of rest (Tsujino et al., 2011). The instantaneous field on 11 May 2001 was used for the initial condition of the tide experiment. The test period was 40 days, unless otherwise noted. In this paper, dates are indicated by UTC or the number of days since the start such as "day 40 ". The time step interval is $3 \mathrm{~min}$, following Sect. 4b of Schwiderski (1980).

The seven experiment cases were executed (Table 2). TIDE and NOTIDE were run with eight tidal constituents and without tide, respectively, and are analyzed in this paper. Results of long integration (one year) are also shown for these two cases. The TIDEa1 case with $\alpha=1$ is used for comparison with a case in which that the SAL term is ignored without violating dynamical balances in the basic field as in a conventional scheme. ${ }^{2}$ The M2 case, which uses the M2 constituent only, and the K1 case, which uses the K1 constituent only, are used for dynamical analysis of tides in the model. M2v2 and M2v10, in which the tidal horizontal viscosity is changed to $2 \times 10^{4} \mathrm{~m}^{2} \mathrm{~s}^{-1}$ and $1 \times 10^{5} \mathrm{~m}^{2} \mathrm{~s}^{-1}$, respectively, are used to examine dependency on the $\boldsymbol{D}_{l t}$ setting. Usage of different values for the horizontal viscosity for the tides as opposed to basic fields is based on Polzin (2008),

\footnotetext{
${ }^{1}$ Two misprints were found: the correct astronomical argument of $\mathrm{P} 1$ is $-h_{0}-90$, and the day number from the reference date $D$ is $d+365(y-1975)+\operatorname{Int}((\mathrm{y}-1973) / 4)$.

${ }^{2}$ Since $\boldsymbol{D}_{l t}$ differs from $\boldsymbol{D}_{b}$, this case does not correspond perfectly to a case using the conventional scheme where tidal and basic fields are treated without separation.
} 
(a) $\eta$ (TIDE)

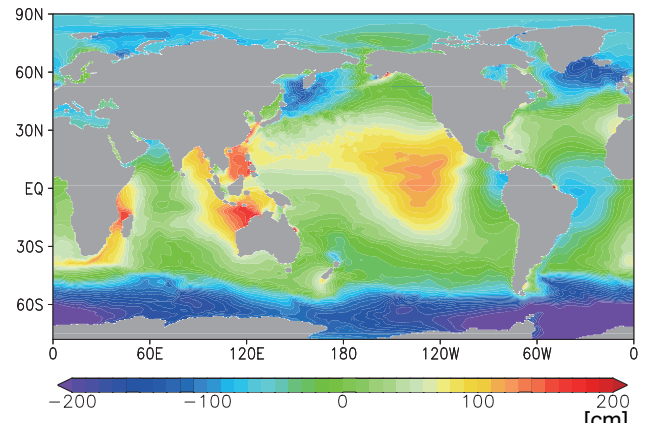

(c) $\eta_{\text {It }}$ (TIDE)

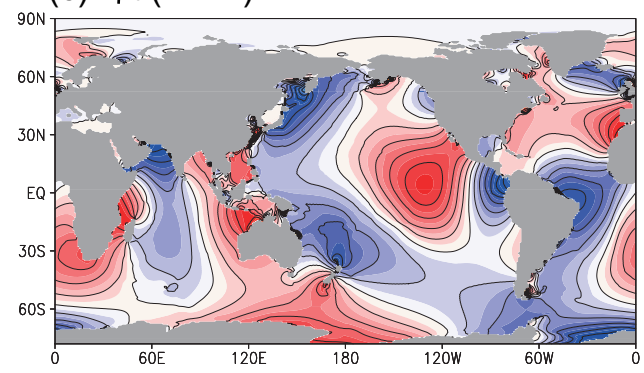

(e) $\eta_{\mathrm{t}}^{\mathrm{a}}$

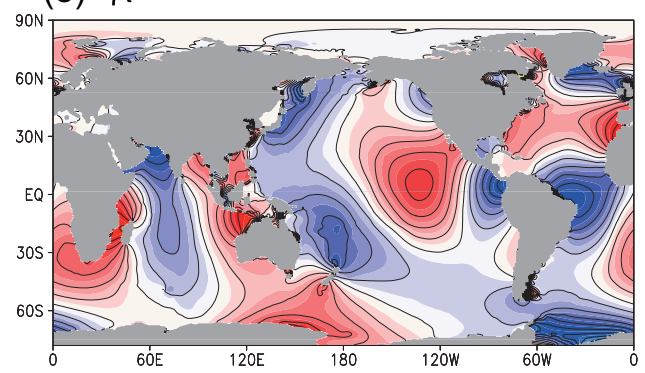

(b) $\eta_{t}$ (TIDE)

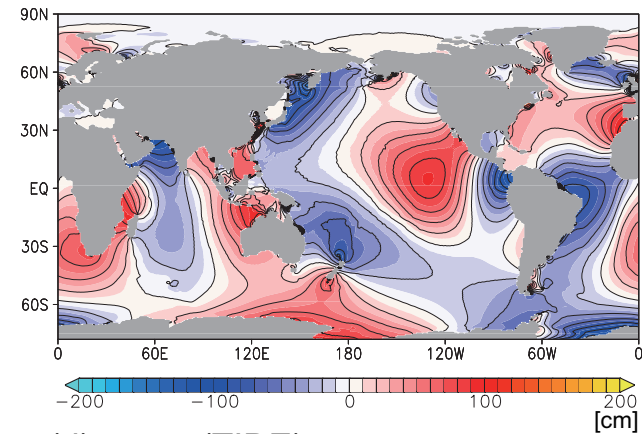

(d) $\eta_{\mathrm{t}}-\eta_{\mathrm{It}}(\mathrm{TIDE})$

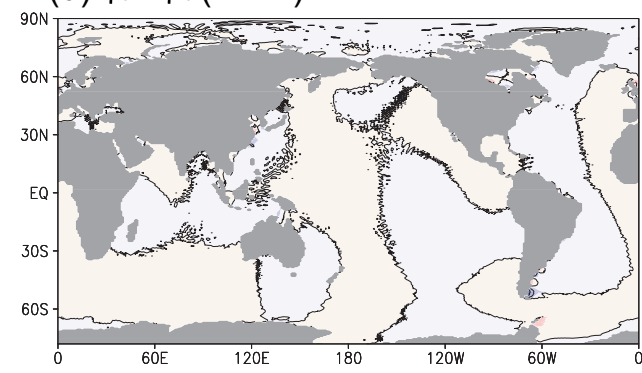

(f) $\eta_{\mathrm{t}}(\mathrm{TIDEa} 1)$

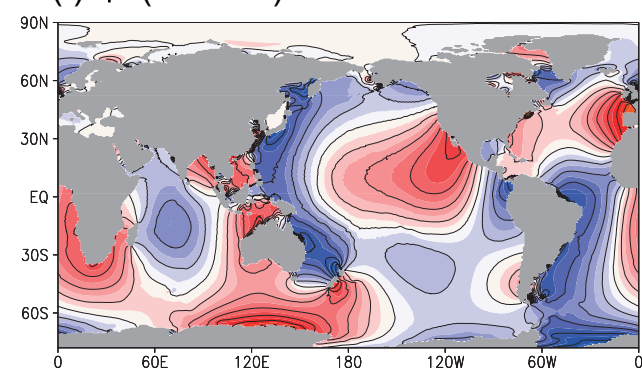

Fig. 2. (a) SSH $\eta$, (b) tidal height $\eta_{t}$, (c) height of the linear tidal component $\eta_{l t}$, (d) the difference $\eta_{t}-\eta_{l t}$ in case TIDE, (e) data assimilation analysis $\eta_{t}^{a}$ (Matsumoto et al., 2000) and (f) $\eta_{t}$ in TIDEa1 at the end of the 40-day experiments (20 June 2001 00:00 UTC). The same colors are used in (b-f); red indicates ascend (positive), whereas blue indicates descend (negative). The contour interval is $20 \mathrm{~cm}$.

who proposed an interpretation of horizontal viscosity as a way to parameterize the interaction between mesoscale eddies and internal waves. That is, the usage of different values means that mesoscale eddies interact differently with internal waves that result from geostrophic adjustment than with internal tides. The experimental period was 10 days in M2, $\mathrm{K} 1, \mathrm{M} 2 \mathrm{v} 2$ and M2v10.

In this paper, we analyzed the tidal height $\eta_{t}$ defined by the SSH anomaly from NOTIDE,

$\eta_{t} \equiv \eta-\eta$ (NOTIDE).

It should be noted that this is in order to investigate precisely the tidal field including secondary oscillations in the model. Actually, performing two simulations (one with and one without tides) is not required in order to obtain the tidal heights with some accuracy. Simulation output of the linear tidal component, $\eta_{l t}$, may serve as a basic data set of tides in an OGCM, since this component represents most of the tidal variations as shown in Sect. 3.1. Alternatively, based on output of $\eta$, the tidal heights can be estimated by the anomaly from the 25-hour running mean, or the deviation from the dynamic heights after Arbic et al. (2010) (density data is needed in this case).

The global tide data set of Matsumoto et al. (2000) (NAO.99b) was downloaded from the web (http://www. miz.nao.ac.jp/staffs/nao99/index_En.html) and used to assess model reproducibility of the tidal height. This data set is a reanalysis product made by the assimilation of SSH satellite observations to a barotropic tide model with a horizontal resolution of $0.5^{\circ}$. Though they reported that errors in tidal height are as much as $2 \mathrm{~cm}$ at maximum (Figs. 3 and 4 of their paper), the data set is assumed to represent the actual tidal heights in this study. 
Table 3. The RMS error of the tidal height $(\mathrm{cm})$ and the percentage of SSH variance captured (\%) in TIDE and TIDEa1. The values of "TIDE(coastal)" are calculated in the region shallower than $1000 \mathrm{~m}$.

\begin{tabular}{lccc}
\hline Case & TIDE & TIDEa1 & TIDE(coastal) \\
RMS error $(\mathrm{cm})$ & 10.0 & 31.3 & 35.6 \\
percentage of SSH $(\%)$ & 90 & 2 & 42 \\
variance captured & & & \\
\hline
\end{tabular}

\section{Results}

\subsection{Tidal height}

The test experiments with our tidal scheme successfully reproduced many of the large-scale features known to be in the tidal field as well as basic field. Figure $2 \mathrm{a}$ shows the instantaneous field of SSH $\eta$ at the end of case TIDE. Tides with a basin scale are clearly seen, along with the geostrophic circulation on a large scale, (e.g. the meridional gradient of the Antarctic Circumpolar Current).

Figures $2 \mathrm{~b}$ and $2 \mathrm{c}$ show the tidal heights, $\eta_{t}$, and the linear tidal component of SSH, $\eta_{l t}$, respectively, in TIDE. As noted in Sect. 2.2, the former represents the whole tidal motion, including nonlinear effects, while the latter is the primary barotropic response to the tidal forcing following Eqs. (11) and (12). Except for a few differences in coastal areas $(10 \mathrm{~cm}$ at maximum), they are almost identical globally (Fig. 2d). This result supports our expectation that the linear tidal components, $\boldsymbol{U}_{l t}$ and $\eta_{l t}$, represent most of the tidal motions. In our new scheme, only the linear tidal equations adopt parameterizations specified for tides; here, we suggest that this is enough to reproduce tides in a global model.

For comparison, Fig. 2e shows the tidal height of the reanalysis data set (Matsumoto et al., 2000), $\eta_{t}^{a}$. The patterns of sea surface elevation in $\eta_{t}$ (or $\eta_{l t}$ ) of TIDE and $\eta_{t}^{a}$ are very similar in the Indian, Pacific and Atlantic oceans, though there are some differences around the Antarctic continent. The amplitudes of these variables are also very similar. For example, the local maximum in the eastern equatorial Pacific region is approximately $87 \mathrm{~cm}$ in both $\eta_{t}$ and $\eta_{t}^{a}$. This result suggests that our new scheme worked as expected, and that the model reproduced a realistic time evolution of the tides.

By contrast, $\eta_{t}$ in TIDEa1, which ignored the SAL term, is different from $\eta_{t}^{a}$ (Fig. 2f). For example, the local maximum in the eastern Pacific was not located in the equatorial region, but adjacent to the west coast of North America, and the pattern of sea surface elevation around New Zealand deviated counterclockwise by approximately $60^{\circ}$. The contrasting results between TIDE and TIDEa1 indicate that realistic tides cannot be modeled in an OGCM if the original barotropic equation is used to calculate time evolution of tides. It is necessary to use parameterizations developed for tides. Even the scalar approximation for the SAL term, for example, improves simulations of the tides.
To investigate the causes of the differences between TIDE and TIDEa1 in detail, the amplitude of the tidal height variation is evaluated by the root mean square of $\eta_{t}, \eta_{\mathrm{RMS}}$,

$$
\eta_{\mathrm{RMS}}=\left(\frac{1}{T_{1}-T_{0}} \int_{T_{0}}^{T_{1}} \eta_{t}^{2} d t\right)^{1 / 2}
$$

where $T_{0}$ and $T_{1}$ indicate 5 May 2001 and 20 June 2001, i.e. the times after 10 and 40 days from the experiment start, respectively. Figure 3 shows $\eta_{\text {RMS }}$ in TIDE, TIDEa1 and the assimilation data set. Comparing $\eta_{\mathrm{RMS}}$ (TIDE) and the assimilation result $\eta_{\mathrm{RMS}}^{a}$, the distributions and the local maxima are very similar except for some differences (e.g., $\eta_{\text {RMS }}$ is slightly smaller in the Indian Ocean, and larger in the western equatorial Pacific region). Similarly, $\eta_{\mathrm{RMS}}(\mathrm{TIDEa} 1)$ is close to $\eta_{\mathrm{RMS}}^{a}$, though its distribution seems somewhat distorted. The means by which the viscosity parameterization influences the performance of the tidal amplitude is discussed in Sect. 3.2.

Next, the reproducibility of the tidal phase is examined. As representative results, Fig. 4 shows time series of $\eta_{t}$ at three locations in the equatorial Pacific, the equatorial Atlantic and the central Indian Ocean. In contrast to the amplitudes, the reproducibility of the tidal phase differs substantially between TIDE and TIDEa1. The $\eta_{t}$ phase is ahead by up to $1.5 \mathrm{~h}$ from $\eta_{t}^{a}$ in TIDEa 1 at all the locations (corresponding to $45^{\circ}$ for semi-diurnal tides). On the other hand, $\eta_{t}$ is in phase with $\eta_{t}^{a}$ in TIDE, as the phase differences are found to be less than $0.5 \mathrm{~h}$. As a result, the difference between $\eta_{t}$ and $\eta_{t}^{a}$ decreases drastically in TIDE, in comparison with TIDEa1, nearly everywhere. Thus, the problem of the tidal phase being too far ahead in TIDEa1 is corrected to some extent in TIDE. This is one important reason for the difference in the reproducibilities of the two cases shown in Fig. 2:

A possible mechanism of this correction is as follows. Introduction of the SAL term modifies the gravitational acceleration to $\alpha g$ virtually in TIDE, as indicated by Eq. (20). Since $\alpha$ is less than unity ( 0.88 in our settings), the phase velocity of shallow gravitational waves $(\sqrt{\alpha g / H})$ becomes slower. This mechanism may contribute to reproducibility of the tidal phase.

The reproducibility of the tidal height is evaluated quantitatively here. For this purpose, a root-mean-squared error of $\eta_{t}, \eta_{\mathrm{RMSE}}$, is calculated using $\eta_{t}^{a}$ as a reference (Fig. 5),

$$
\eta_{\mathrm{RMSE}} \equiv\left(\frac{1}{T_{1}-T_{0}} \int_{T_{0}}^{T_{1}}\left(\eta_{t}-\eta_{t}^{a}\right)^{2} d t\right)^{1 / 2} .
$$

In TIDE, $\eta_{\mathrm{RMSE}}$ is less than $20 \mathrm{~cm}$ - even in the open oceans where $\eta_{\text {RMS }}$ is large - and less than $10 \mathrm{~cm}$ in most other regions, except for the Southern Ocean south of the polar convergence. Meanwhile, in TIDEa1, $\eta_{\text {RMSE }}$ is more than $20 \mathrm{~cm}$ 
(a) $\eta_{\mathrm{RMS}}(\mathrm{TIDE})$

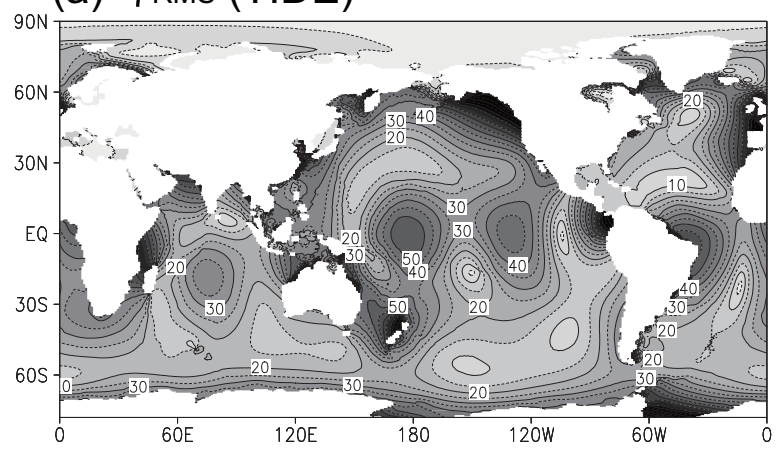

(b) $\eta_{\mathrm{RMS}^{\mathrm{a}}}$

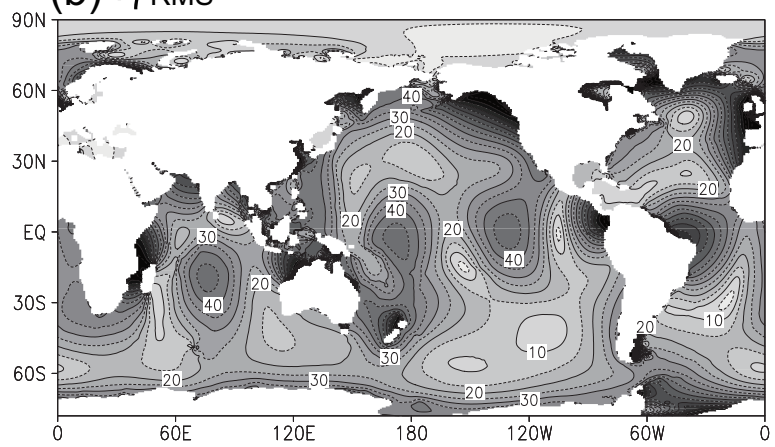

(c) $\eta_{\text {RMS }}($ TIDEa1)

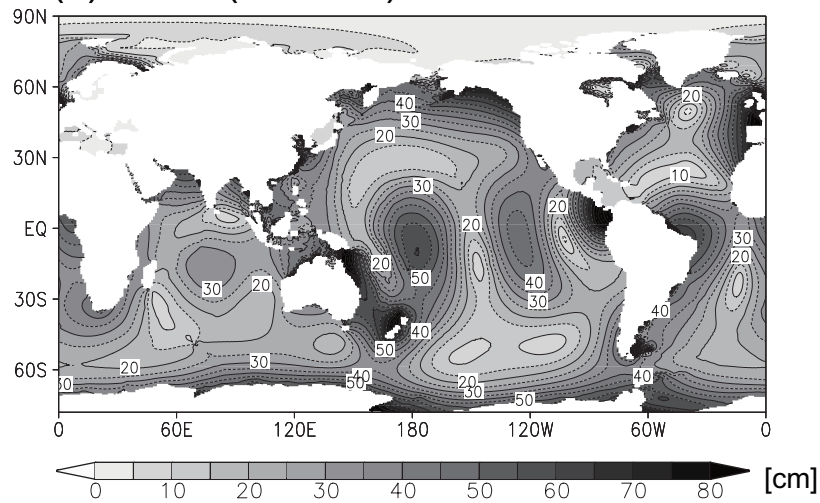

Fig. 3. Root mean square of tidal height, $\eta_{\mathrm{RMS}}$, (Eq. 24) in (a) TIDE, (b) assimilation analysis and (c) TIDEa1. The contour interval is $5 \mathrm{~cm}$.

in most regions such that it reaches values comparable to $\eta_{\text {RMS itself. }}$

Following Arbic et al. (2004), who developed a highlytuned two-layer tide prediction model without a data assimilation technique, the root-mean-squared error is averaged over the region ranging from $66^{\circ} \mathrm{S}$ to $66^{\circ} \mathrm{N}$ with water depth exceeding $1000 \mathrm{~m}$, which is indicated by $A$,

$\bar{\eta}_{\mathrm{RMSE}} \equiv\left(\frac{1}{A} \iint_{A} \eta_{\mathrm{RMSE}}^{2} d x d y\right)^{1 / 2}$,
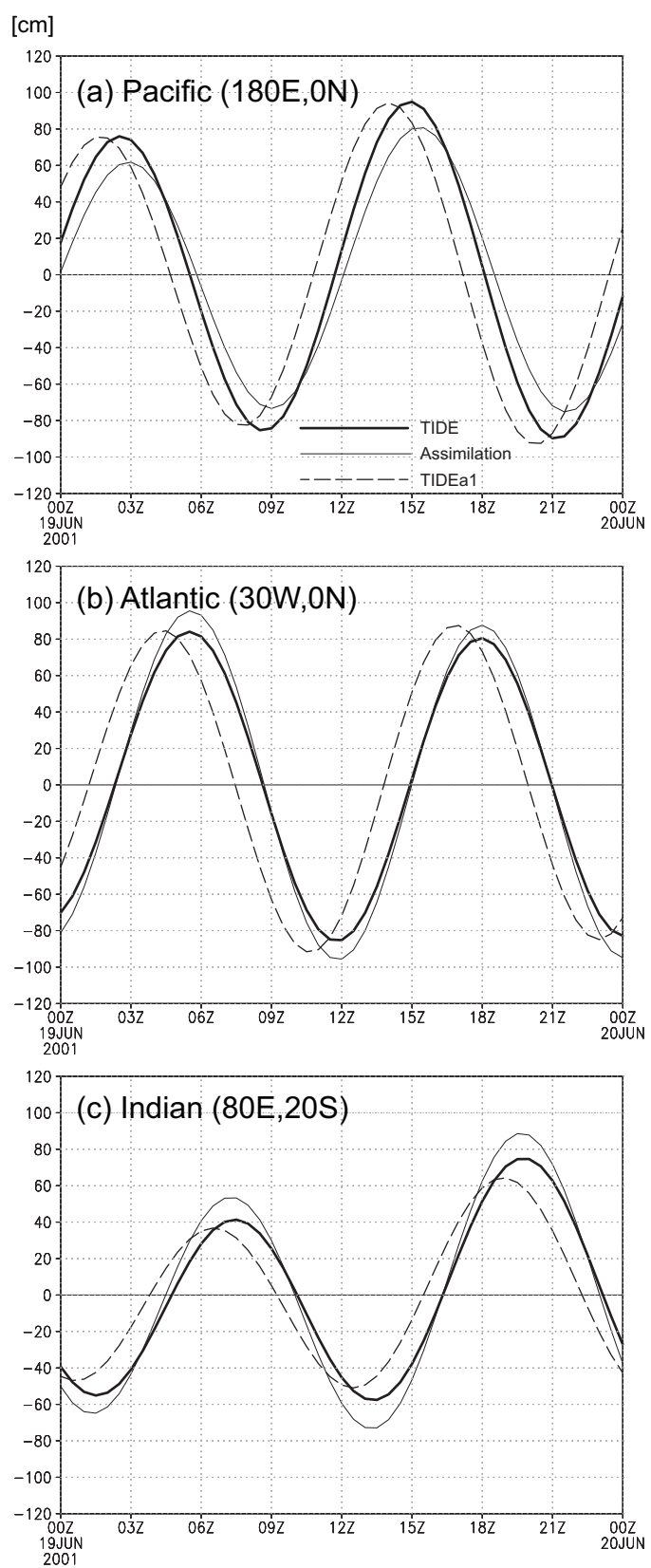

Fig. 4. Time variation of tidal height $\eta_{t}$ [cm] for 19-20 June 2001 (day 40) in (a) the equatorial Pacific $\left(180^{\circ} \mathrm{E}, 0^{\circ} \mathrm{N}\right)$, (b) the equatorial Atlantic $\left(30^{\circ} \mathrm{W}, 0^{\circ} \mathrm{N}\right)$, and (c) the central Indian Ocean $\left(80^{\circ} \mathrm{E}\right.$, $20^{\circ} \mathrm{S}$ ). The thick, thin, and dashed lines indicate TIDE, assimilation analysis, and TIDEa1, respectively.

where $x$ and $y$ are longitude and latitude, respectively. The RMSE in the SSH is as high as $31.3 \mathrm{~cm}$ in TIDEal and $10.0 \mathrm{~cm}$ in TIDE (Table 3). This is comparable to the RMSE found in previous studies (e.g. Jayne and St. Laurent, 2001; Arbic et al., 2004). In addition, Arbic et al. (2004) defined "a percentage of SSH variance captured" by 1 $\left(\bar{\eta}_{\mathrm{RMSE}} / \bar{\eta}_{\mathrm{RMS}}^{a}\right)^{2}$, where $\bar{\eta}_{\mathrm{RMS}}^{a}=31.8 \mathrm{~cm}$ is $\eta_{\mathrm{RMS}}^{a}$ averaged 
(a) $\eta_{\text {RMSE }}(\mathrm{TIDE})$

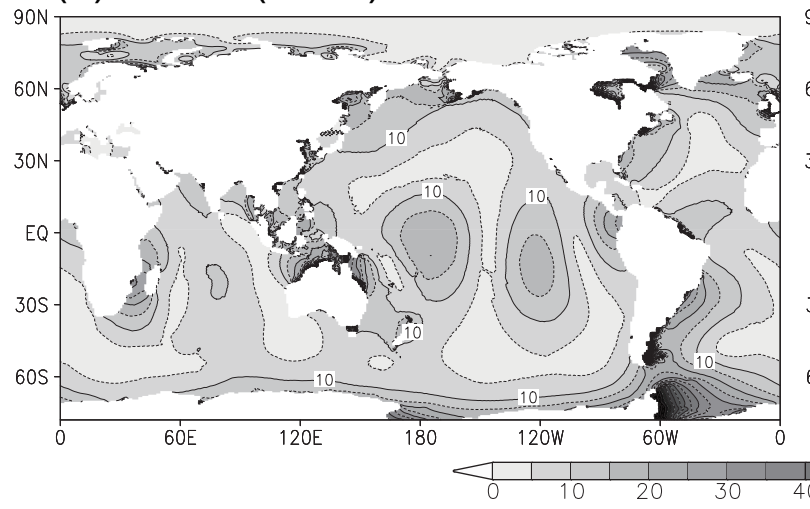

(b) $\eta_{\text {RMSE }}($ TIDEa1)

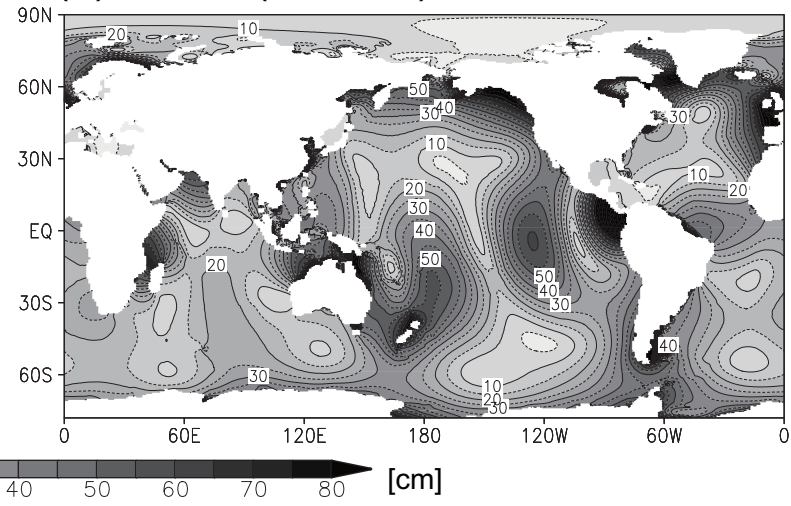

Fig. 5. Root mean square error of the tidal height, $\eta_{\mathrm{RMSE}}$, in (a) TIDE and (b) TIDEa1. The contour interval is $5 \mathrm{~cm}$.

over $A$. The values are $90 \%$ in TIDE, $2 \%$ in TIDEa1, and $92 \%$ in Arbic et al. (2004). The tide reproducibility is very low in TIDEa1, whereas in TIDE - due to taking into account an approximation of the SAL term - it increases to the same level as a tuned tide model. Since a simple viscosity parameterization was used in the experiment, the reproducibility could increase further by adopting more sophisticated parameterizations or tuning the settings more carefully. Though this task is beyond the scope of this paper, some case studies discussed in Sect. 3.2 indicate that the tide reproducibility significantly depends on the viscosity settings.

To simulate circulations affected by tides, it is important to introduce tides realistically into an OGCM, even in coastal areas (e.g. Moon et al., 2010; Kurapov et al., 2010). However, the reproducibility of the tidal heights in coastal areas tended to decrease in our low-resolution model. The root-meansquared error $\eta_{\text {RMSE }}$ averaged over the region shallower than $1000 \mathrm{~m}$ is found to be $35.6 \mathrm{~cm}$, which is three times as large as the $10.0 \mathrm{~cm} \eta_{\mathrm{RMSE}}$ in the open ocean, and the percentage of SSH variance captured is only $42 \%$ (Table 3 ). This is likely because our low-resolution model cannot represent relatively small physical processes affecting coastal tides, such as excitement of internal tides over shelf slopes, waves breaking on shelves, and the friction of complicated topographies (Xing and Davies, 1997; Osborne et al., 2011; Nagai and Hibiya, 2012). Improvement of the coastal tides by the incorporation of our scheme into a high-resolution OGCM is project to be completed in the future.

To verify that an OGCM with our tidal scheme runs stably, long time variations are analyzed here. First, the tidal heights $\eta_{t}$ (TIDE) for 8 days are shown in Fig. 6a at the same location as Fig. 4a, together with $\eta_{t}^{a}$. Amplitude modulation induced by neap and spring tides is found to be well reproduced in the model. Figure $6 \mathrm{~b}$ shows the variation range of $\eta$ (TIDE) for one year at the same location. Quasi-stationary undulation of $\mathrm{SSH}$ is maintained for one year with fortnightly modulation. These results were obtained at the locations of Fig. $4 \mathrm{~b}$ and c, too (not shown). As systematic indices of the model stability, the volume-averaged kinetic energy of the barotropic currents, $\overline{\mathrm{BKE}}$, and the counterpart of the linear tidal currents, $\overline{\mathrm{BKE}}_{l t}$, are calculated by

$$
\begin{aligned}
& \overline{\mathrm{BKE}}=\frac{1}{V} \iiint_{V} \frac{1}{2}\left|\frac{\boldsymbol{U}}{H+\eta}\right|^{2} d x d y d z, \\
& \overline{\mathrm{BKE}}_{l t}=\frac{1}{V} \iiint_{V} \frac{1}{2}\left|\frac{\boldsymbol{U}_{l t}}{H+\eta}\right|^{2} d x d y d z,
\end{aligned}
$$

where $V$ indicates the whole region of the model $\left(\mathrm{m}^{3}\right)$. Monotonic increase or decrease of energy is not found in the two time series of one year, though fortnightly variation exists (Fig. 6c). It can be concluded that the model runs stably with our tidal scheme at least for one year.

\subsection{Tidal motion}

In this subsection, tidal motions reproduced by the new tidal scheme are validated using the results of cases M2 and K1. As shown in Fig. 2, most of the tidal height variation was represented by the linear tidal component $\eta_{l t}$ in our global model. Similarly, barotropic currents with tidal frequencies were almost completely represented by the linear tidal component $\boldsymbol{U}_{l t}$, and therefore $\boldsymbol{U}_{l t}$ is used to compare with past tide studies. Hereafter, the 100-hour experimental results from 20:00 on day 6 (indicated by $T_{0}$ ) to 24:00 on day 10 $\left(T_{1}\right)$ are used for analysis.

As a first step to validate the tidal currents in cases M2 and K1, Fig. 7 shows the mean speed distributions of the barotropic tidal currents, ${\overline{\left|\boldsymbol{u}_{l t}\right|}}^{t}$, calculated by

$$
{\overline{\mid \boldsymbol{u}_{l t}}}^{t}=\frac{1}{T_{1}-T_{0}} \int_{T_{0}}^{T_{1}}\left|\frac{\boldsymbol{U}_{l t}}{H+\eta}\right| d t .
$$




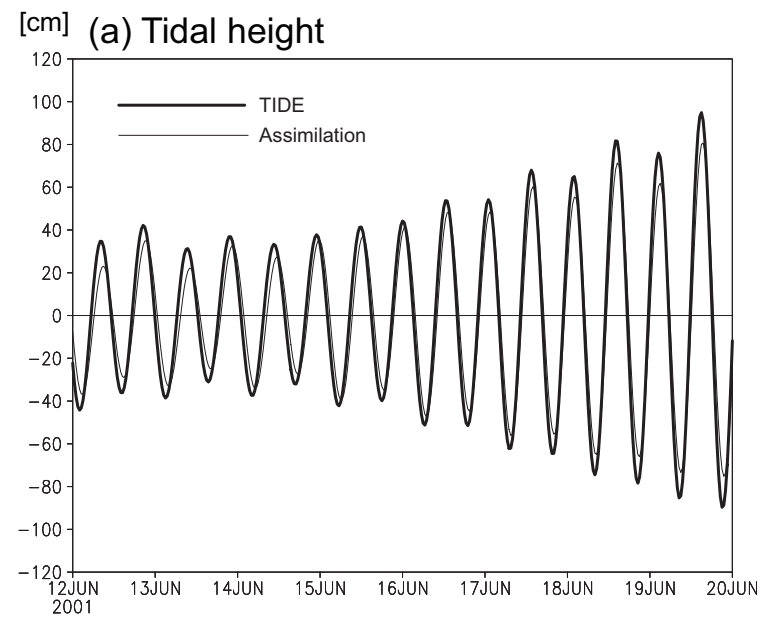

(b) $\mathrm{SSH}$

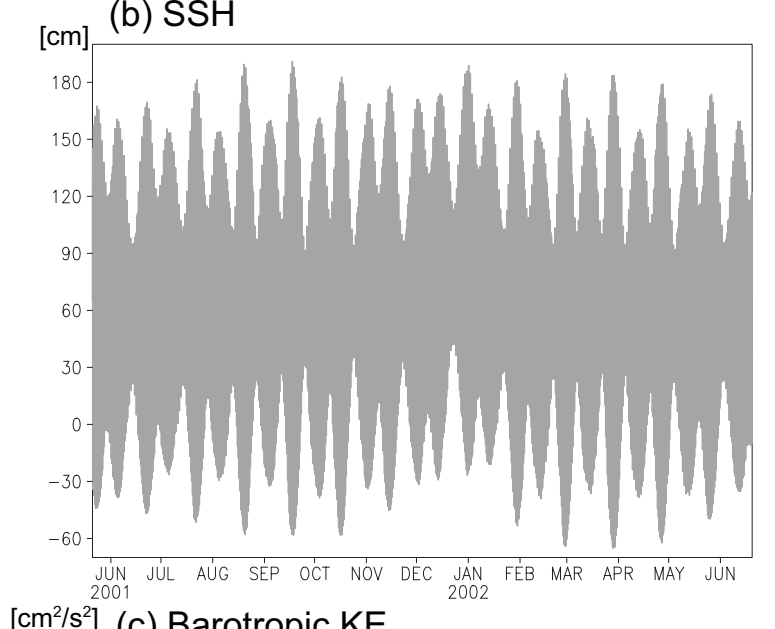

$\left[\mathrm{cm}^{2} / \mathrm{s}^{2}\right]$ (c) Barotropic KE

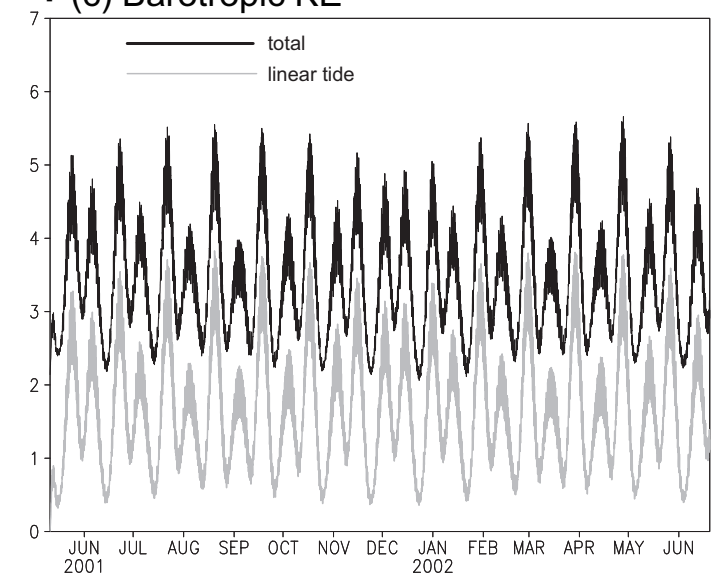

Fig. 6. (a) Same as Fig. 4, but for 12-20 June 2001 (day 32-40). The thick and thin lines indicate TIDE and assimilation analysis, respectively. (b) The variation range of SSH at the site $\left(180^{\circ} \mathrm{E}, 0^{\circ} \mathrm{N}\right)$ over one year - from 21 May 2001 to 20 June 2002 (day 10-405) - in TIDE. (c) Same as (b) but for the volume-averaged barotropic kinetic energy $\left[\mathrm{cm}^{2} \mathrm{~s}^{-2}\right]$ of $\boldsymbol{U}\left(\overline{\mathrm{BKE}}\right.$, black) and $\boldsymbol{U}_{l t}\left(\overline{\mathrm{BKE}}_{l t}\right.$, gray).
For both the M2 and $\mathrm{K} 1$ cases, the tidal currents are strong in coastal areas, especially near Great Britain, Ireland and eastern Asia. In the M2 case, $\left|\overline{\boldsymbol{u}}_{l t}\right| t$ is large over the Mid-Atlantic Ridge, and in the equatorial Pacific. In the K1 case, ${\overline{\mid \boldsymbol{u}_{l t}}}^{t}$ is large in the Indian Ocean and the North Pacific. These results agree well with Fig. 1 in Müller et al. (2010).

Next, we executed an energy analysis for the M2 tide. Generally, the tide energy is supplied to interior ocean regions, and then transported to narrow coastal regions to be dissipated there. Egbert and Ray (2003) analyzed the pathways of the M2 tide energy based on an assimilation model. Following them, the tide energy flux, $\boldsymbol{P}$, and the energy supply, $W$ (i.e., the work which the tidal forcing does on the ocean), are estimated for the linear tidal component using

$$
\begin{aligned}
\boldsymbol{P} & =\frac{1}{T_{1}-T_{0}} \int_{T_{0}}^{T_{1}} \boldsymbol{U}_{l t} \eta_{l t} d t \\
W & =\frac{1}{T_{1}-T_{0}} \int_{T_{0}}^{T_{1}} \boldsymbol{U}_{l t} \cdot \nabla\left(\beta \eta_{0}+\eta_{\mathrm{SAL}}\right) d t .
\end{aligned}
$$

Assuming a steady energy state, the energy dissipation, $D$, can be estimated from the energy balance as

$0=W-\nabla \cdot \boldsymbol{P}-D$.

See Sect. 3.1 of Egbert and Ray (2001) for the derivation of these equations. Figure 8 verifies that the energy was supplied in interior regions, transported to coastal regions, and dissipated there. In addition, the $\boldsymbol{P}$ vector map agrees well with Egbert and Ray (2001). These results indicate that our model reproduced both the tidal heights and the tidal dynamics such as the tidal currents and the energy flux, which are important for effects on the basic fields. Figure $8 \mathrm{~b}$ shows that the tidal energy dissipates over rough topographies such as the Izu-Ogasawara Ridge, the Hawaiian Ridge and the Central Atlantic Ridge, as well as in coastal regions. This likely reflects barotropic-to-baroclinic conversion resulting from excitement of internal tides over rough topographies (see Sect. 3.3).

It has been shown that the precision of a tide model depends primarily on settings of viscosity and friction to dissipate tidal currents (Arbic et al., 2004). In our model experiments, tides also significantly depended on the viscosity settings, especially the horizontal viscosity, $v$. Figure 9 shows $\eta_{t}$ in the two additional cases where $v$ was decreased to $2 \times 10^{4} \mathrm{~m}^{2} \mathrm{~s}^{-1}$ (case M2v2) and increased to $10 \times 10^{4} \mathrm{~m}^{2} \mathrm{~s}^{-1}$ (M2v10), together with the standard case with $v=6 \times 10^{4}$ $\mathrm{m}^{2} \mathrm{~s}^{-1}$. Though the patterns of sea surface elevation were similar, the magnitudes differed significantly, e.g., the local maxima in the eastern equatorial Pacific of $84 \mathrm{~cm}, 63 \mathrm{~cm}$ and $52 \mathrm{~cm}$ in cases M2v2, M2 and M2v10, respectively. In addition, it was revealed that the amplitudes of tidal height variation were mainly controlled by the horizontal viscosity 
(a) M2

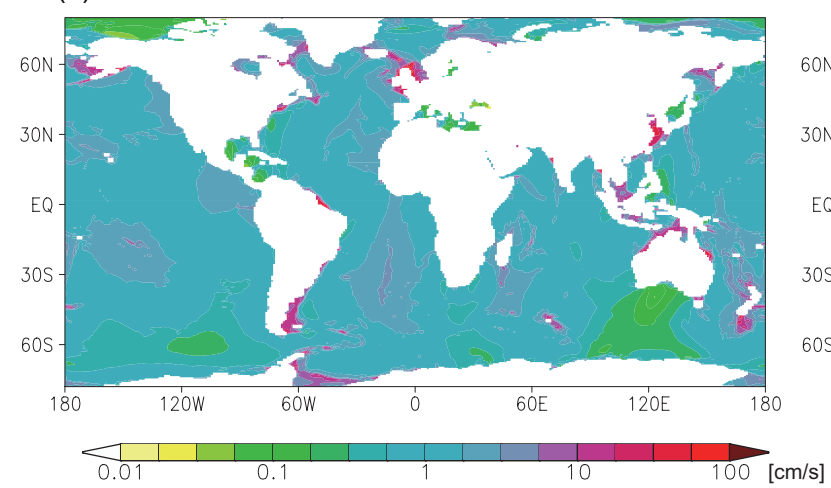

(b) K1

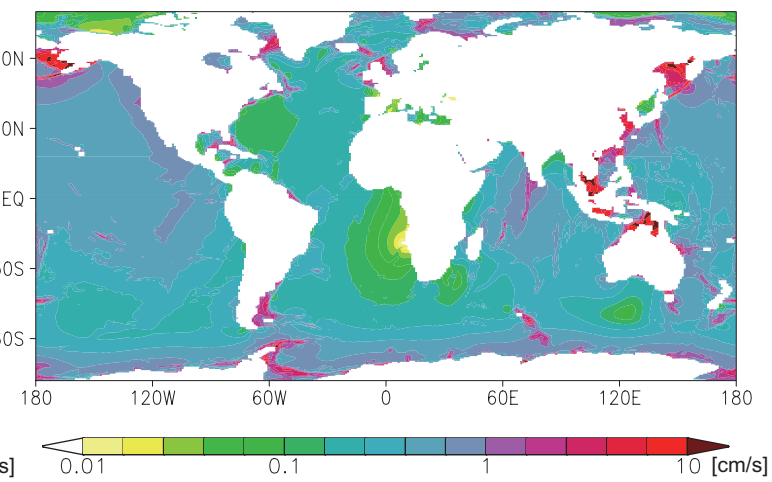

Fig. 7. Speed of the barotropic tidal currents ${\overline{\left|\boldsymbol{u}_{l t}\right|}}^{t}$ of (a) M2 tide and (b) K1 tide averaged over 100h from 20:00 on day 6 to 24:00 on day 10. The colors are the same as in Fig. 1 of Müller et al. (2010), and the units are $\mathrm{cm} \mathrm{s}^{-1}$.

(a) W, P

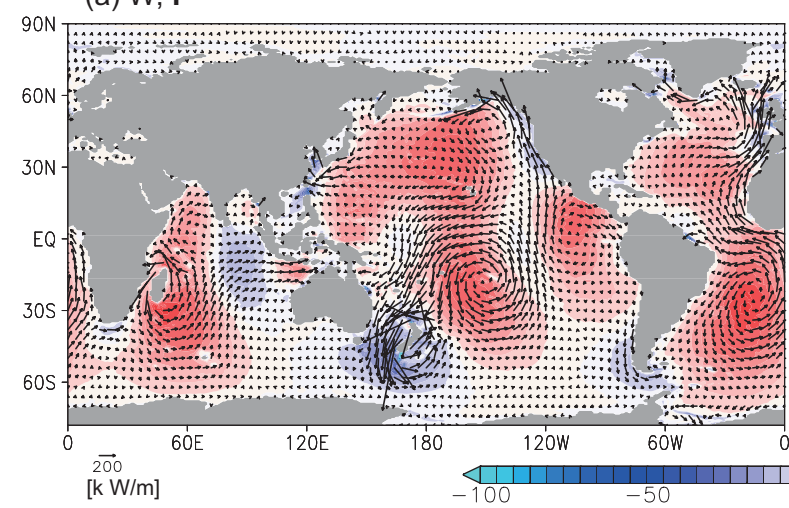

(b) $-\mathrm{D}$

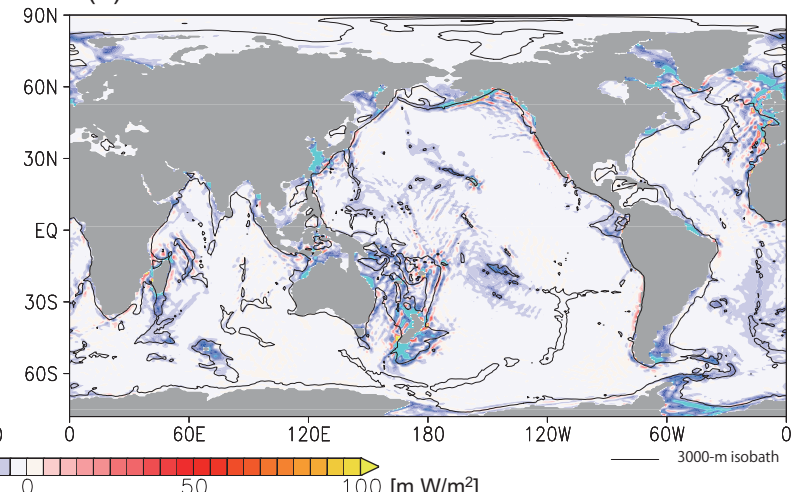

Fig. 8. (a) Tidal energy flux $\boldsymbol{P}$ (vector) and power on the ocean $W\left(10^{-3} \mathrm{Wm}^{-2}\right)$ (colored shading) in case M2. The unit length of the vectors is $200 \mathrm{k} \mathrm{Wm}^{-1}$ the same as Fig. 1 of Egbert and Ray (2001). (b) Tidal energy dissipation $-D$ in case M2. The $3000 \mathrm{~m}$ isobath is shown to illustrate the bottom topography.

between the water and the lateral boundary of bottom topography, rather than the viscosity of the interior currents or bottom friction (not shown). These results are consistent with Schwiderski (1980) and Arbic et al. (2004), who reported that interaction between tidal currents and bottom topography is one of the most important processes in dissipation of tides. As noted here, by using the new scheme, we could adjust the tide viscosity and friction parameters, including interaction between tides and topography, without altering the original OGCM equations. This feature is essential to the realistic introduction of tides into an OGCM, as Arbic et al. (2010) pointed out.

\subsection{Effects on basic fields}

As noted in Sect. 2.2, our new tidal scheme is designed so that interaction processes between the basic fields and tides are represented in the original OGCM framework. Owing to this, the tidal effects on the basic fields would be reproduced naturally in the model, as long as the tidal currents are gen- erated realistically by the scheme. Therefore, we expected that some changes would occur in the velocity and tracer fields of the test experiments, since the tidal currents were well reproduced. In order to validate impacts of our tidal scheme, changes in the basic fields are summarized briefly in this subsection, though the experimental period (40 days) and the model resolution $\left(1^{\circ} \times 1 / 2^{\circ}\right)$ are not enough to represent thorough modification of the basic fields in the real ocean.

In general, active excitement of internal waves is one of the main impacts of tides on the basic fields. Figure 10 shows vertical velocity $w$ at the depth of $1900 \mathrm{~m}$ in cases TIDE and NOTIDE. In NOTIDE, with the exception of the equatorial region, $w$ was $\mathrm{O}\left(10^{-3}\right) \mathrm{cm} \mathrm{s}^{-1}$, while $w$ in TIDE was more than $10^{-2} \mathrm{~cm} \mathrm{~s}^{-1}$ over large areas. This difference indicates excitement of internal tides in TIDE. In fact, the vertical velocity was especially large over rough topographies such as the Emperor Seamounts (near $170^{\circ} \mathrm{E}$ ), the Hawaiian Ridge, and the Izu-Ogasawara Ridge $\left(140^{\circ} \mathrm{E}\right)$, suggest- 
(a) M2v2 $\left(v=2 . e 4 \mathrm{~m}^{2} / \mathrm{s}\right)$

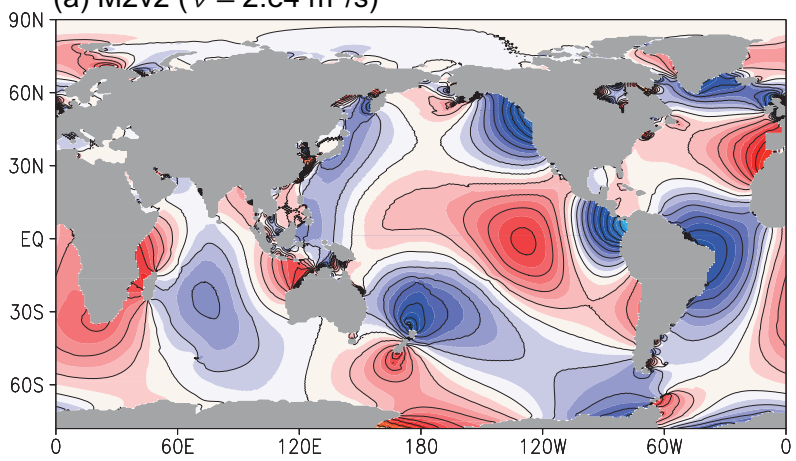

(b) M2 (6.e4 m²/s)

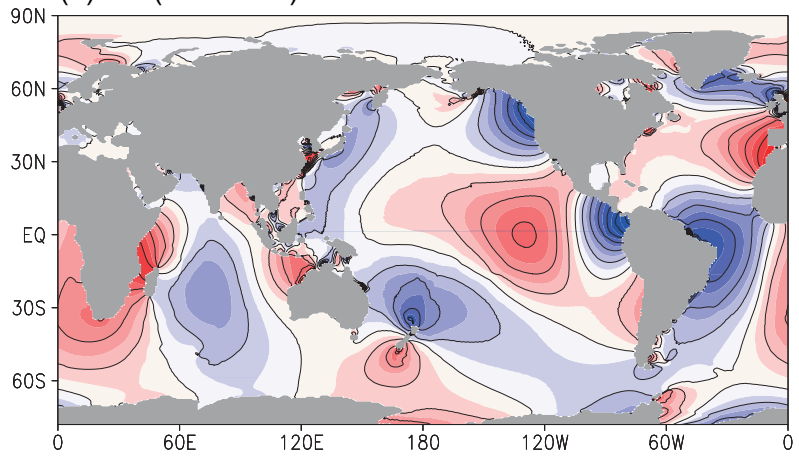

(c) M2v10 (10.e4 m²/s)

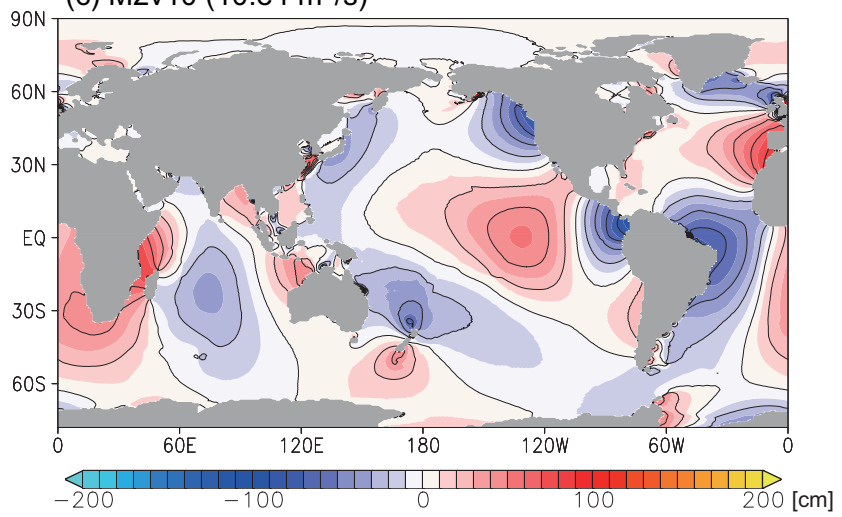

Fig. 9. Tidal height $\eta_{t}$ at the end of the 10-day experiments (00:00 on 21 May 2001) in cases (a) M2v2 ( $\left.v=2 \times 10^{4} \mathrm{~m}^{2} \mathrm{~s}^{-1}\right)$, (b) M2 $\left(6 \times 10^{4}\right)$ and $\left(\right.$ c) M2v10 $\left(10 \times 10^{4}\right)$. The contour interval is $20 \mathrm{~cm}$.

ing active excitement due to interaction between tides and topographies. This is consistent with the energy dissipation $D$ in Fig. 8b.

Figure 10 shows that $w$ had a zonal band pattern with a meridional wavelength of approximately $200 \mathrm{~km}$. This pattern is very similar to the result of Komori et al. (2008) (their Fig. 1), who simulated excitement of internal waves by wind using a model with a horizontal resolution of $1 / 4^{\circ}$. However, Arbic et al. (2010) reported that internal tides have a ripplelike pattern spreading from bottom topographies, using an eddy-resolving model with a horizontal resolution of approx- imately $1 / 10^{\circ}$. Since reproducibility of internal tides depends strongly on model resolution (Niwa and Hibiya, 2011), this difference suggests that our model resolution of $1^{\circ} \times 1 / 2^{\circ}$ was not enough to represent internal tides.

To examine how the internal tides are excited in the model in more detail, potential density $\sigma_{0}$ is analyzed around Hawaii, where active excitement is expected from the $w$ distribution (Fig. 10). Figure 11a shows a time variation of $\sigma_{0}$ vertical distribution in TIDE and NOTIDE. No remarkable variation is found in NOTIDE (thin lines), while vertical undulation of the isopycnals with a period of half a day is clearly seen (thick lines). The amplitude reaches $50 \mathrm{~m}$, which means that the model represented heaving of the isopycnals accompanied by internal tides. Figure $11 \mathrm{~b}$ is a Hovmöller diagram showing meridional and temporal change of potential density anomaly $\sigma_{0}^{\prime}$ at the depth of $1000 \mathrm{~m}$ in TIDE, and $\sigma_{0}^{\prime}$ is the anomaly from the time average as

$\sigma_{0}^{\prime}=\sigma_{0}-\frac{1}{T_{1}-T_{0}} \int_{T_{0}}^{T_{1}} \sigma_{0} d t$,

where $T_{0}$ and $T_{1}$ indicate 18 June 2001, 00:00 and 20 June, 00:00, respectively. In Fig. 11b, $\sigma_{0}^{\prime}$ changed most dramatically over the rough topography around Hawaii $\left(\sim 21^{\circ} \mathrm{N}\right)$, showing oscillation with a period of half a day. Subsequently, the anomaly propagated northward and southward with decay (the arrows in the figure). Though the model meridional resolution of $0.5^{\circ}$ is clearly insufficient to represent the spatial structure of the internal tides, it can be concluded that excitement and propagation of the internal tides were represented to some extent.

Fig. 12a shows another impact on the ocean, the sea surface temperature (SST) anomaly, $\Delta \bar{T}^{t}$,

$\Delta \bar{T}^{t}=\bar{T}^{t}(\mathrm{TIDE})-\bar{T}^{t}(\mathrm{NOTIDE})$,

where $\bar{T}^{t}$ indicates the last 25-hour mean temperature (in 19 June). Introduction of tides resulted in a SST decrease of $0.1-0.5^{\circ} \mathrm{C}$ over large areas of the Northern Hemisphere. As shown by a vertical temperature profile (Fig. 12b), the surface layer $(0-15 \mathrm{~m})$ became cooler, while the subsurface layer $(20-40 \mathrm{~m})$ became warmer, and the temperature stratification was weakened. That is, development of the thermocline in subtropical and subpolar regions of the Northern Hemisphere during its summertime was hampered, and, as a result, SST increase in early summer was weakened. This can likely be attributed to the process in which vertical shear in internal tides feeds vertical mixing in the surface layer through the vertical mixing scheme, although the mixing scheme only intermittently predicted large vertical diffusivities. By contrast, $\Delta \bar{T}^{t}$ was relatively small in the Southern Hemisphere's winter. One reason may be that tidal mixing hardly affected the vertical temperature distribution there, since the surface layer was originally well mixed via 


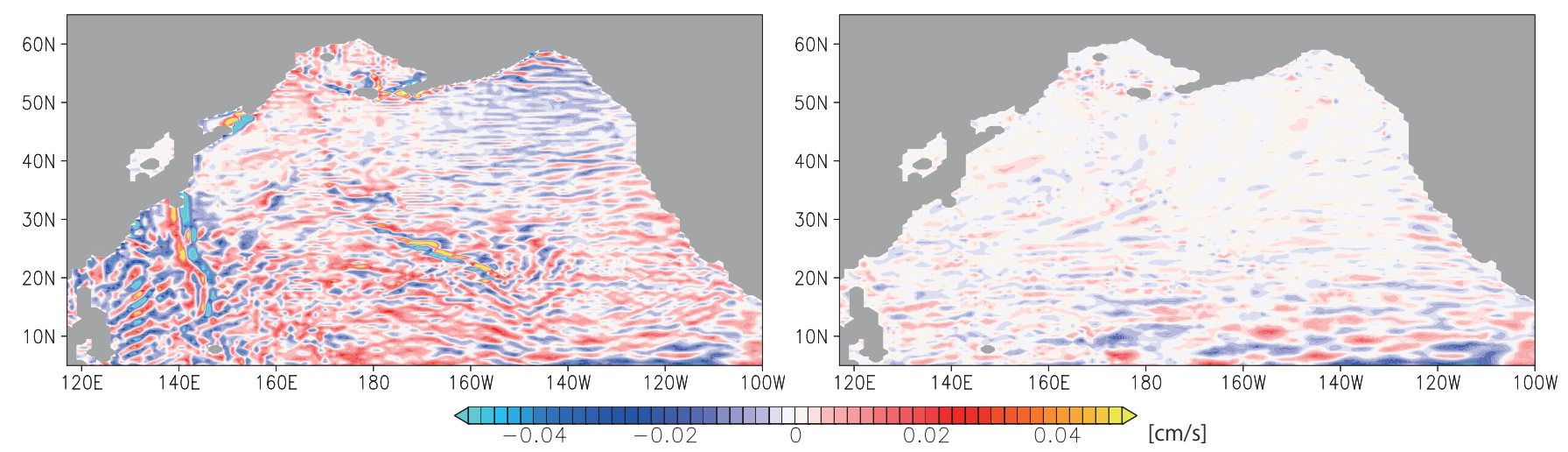

Fig. 10. Vertical velocity $w\left(\mathrm{~cm} \mathrm{~s}^{-1}\right)$ at $1900 \mathrm{~m}$ depth in the North Pacific in (a) TIDE and (b) NOTIDE. The instantaneous distributions at the end of the 40-day experiment are shown.
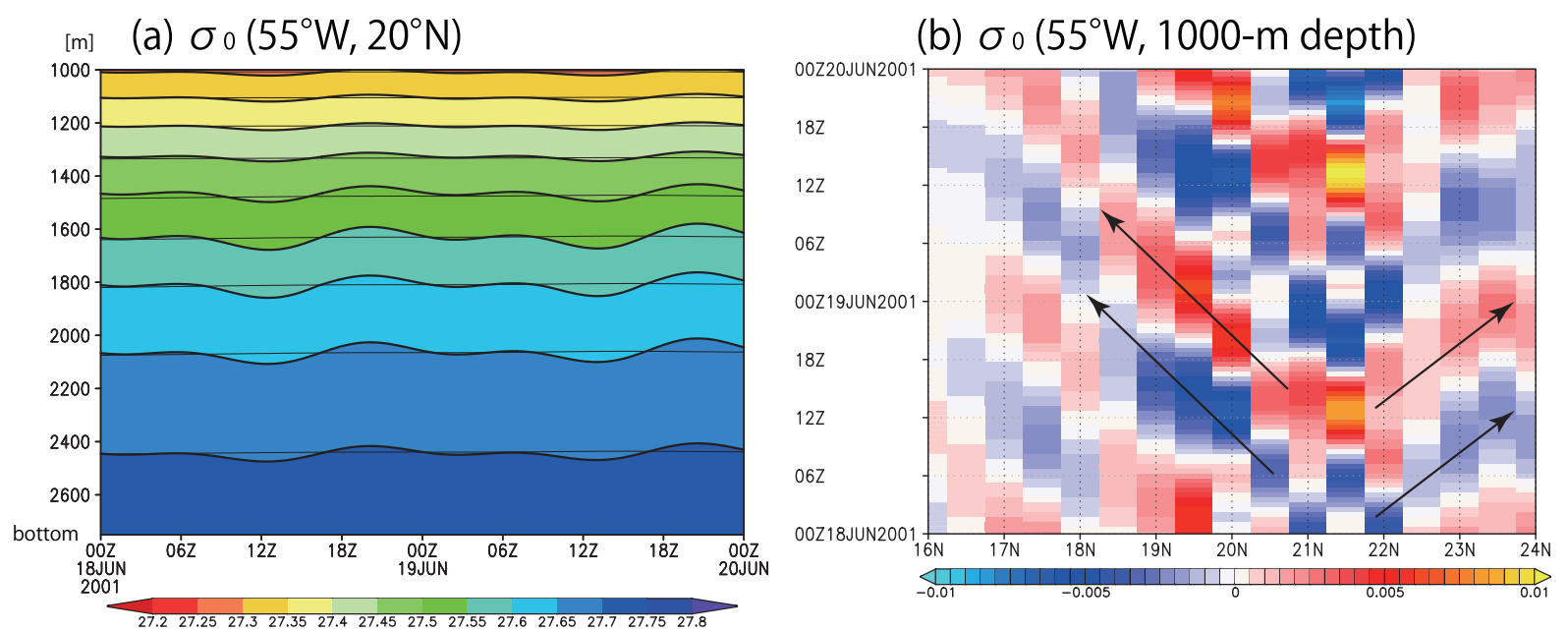

Fig. 11. (a) Vertical distribution of potential density $\sigma_{0}\left(\mathrm{~kg} \mathrm{~m}^{-3}\right)$ from bottom to the $1000 \mathrm{~m}$ depth at $\left(55^{\circ} \mathrm{W}, 20^{\circ} \mathrm{N}\right)$ for $18-20 \mathrm{June} 2001$ (day 39-40) of TIDE (thick lines and colored shading) and NOTIDE (thin lines). (b) Hovmöller diagram of potential density anomaly $\sigma_{0}^{\prime}$ at $55^{\circ} \mathrm{W}, 1000-\mathrm{m}$ depth in TIDE. The meridional range is $16-24^{\circ} \mathrm{N}$, and the time period is the same as (a). The arrows indicate propagation of internal tides.

surface cooling. Both temperature and salinity were almost uniform from the surface to the depth of $80 \mathrm{~m}$. Another reason may be that representation of the tidal mixing was not adequate due to the low resolution of our model. Low resolution is also likely responsible for the fact that large vertical diffusivities were only intermittently predicted in the Northern Hemisphere.

The SST decrease with the inclusion of tides was especially large in shallow coastal regions; e.g., more than $1^{\circ} \mathrm{C}$ around the islands of Great Britain and Ireland. Since this decrease was accompanied with weakening of stratification, the reason is likely that strong tidal currents (Fig. 7) induced vertical mixing through shear instability in the bottom layer, as reported by observational and numerical studies about tidal fronts (Simpson and Hunter, 1974; Müller et al., 2010). The SST anomaly was also large in some polar coastal seas, such as the Greenland Sea and the Ross Sea. This is consistent with the findings of previous studies, which showed significant tidal impacts on dense water formation processes there (Pereira et al., 2002; Robertson, 2001a, b).

Our experiment of 40 days did not show any significant changes in large-scale circulations. The differences between the currents in TIDE and NOTIDE were less than $1 \mathrm{~cm} \mathrm{~s}^{-1}$ in the open oceans, and $10 \mathrm{~cm} \mathrm{~s}^{-1}$ at maximum in coastal areas (not shown). This result is consistent with a study about tidal effects (Bessiéres et al., 2008), which suggests that our tidal scheme did not generate spurious currents. However, considering the report that tidal mixing modified the Atlantic North Current pathway in a long-term integration of a climate model (Müller et al., 2010), tidal impacts on large scale circulations may appear if we run the model much longer. (In our one-year experiment, the velocity difference between 


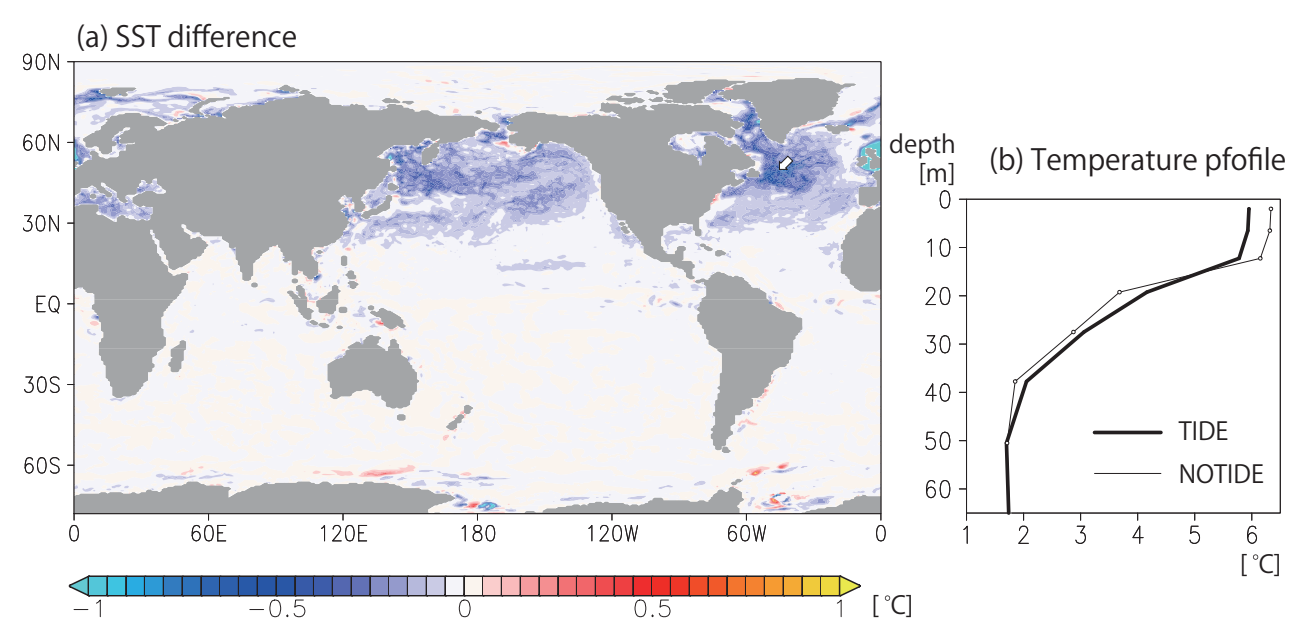

Fig. 12. (a) SST difference between TIDE and NOTIDE $\Delta \bar{T}^{t}\left(\mathrm{C}^{\circ}\right)$. (b) Vertical profiles of temperature $\bar{T}^{t}$ at the site $\left(50^{\circ} \mathrm{W}, 50^{\circ} \mathrm{N}\right)(\mathrm{marked}$ in (a)) in TIDE (thick line) and NOTIDE (thin). The vertical range from surface to $65 \mathrm{~m}$ depth is shown. Both (a) and (b) use 25 -hour averages of the end of the 40-day experiments.

TIDE and NOTIDE reached approximately $10 \%$ of the total velocity in two months. However, it did not expand further after that.)

Though plausible impacts on the OGCM were obtained by our scheme as for impacts of tidal currents, it should be noted that our experiment is the initial investigation of the scheme. In particular, the horizontal resolution of the model is too low to represent internal tides or tidal mixing processes (Matsumoto et al., 2000). With implementation of this scheme in a higher resolution OGCM, evidence of barotropic to baroclinic energy conversion can be provided directly via vertical velocity and potential density, and the conversion rate and coastal effects (Simmons et al., 2004) can be investigated in more detail. A thorough investigation about the process for tidal currents to intensify vertical mixing through velocity shear and turbulence will also be possible. We plan to execute a long-term experiment in order to examine the tidal impacts, including dependencies on model resolution or mixing parameterizations, in more detail.

\section{Conclusions}

A new practical scheme is proposed to introduce tides explicitly into ocean general circulation models (OGCMs). In this scheme, barotropic linear response to the tidal forcing is calculated by the time differential equations modified for ocean tides, instead of the original barotropic equations of the OGCM. This allows for the usage of various parameterizations specified for tides, such as the self-attraction/loading (SAL) effect and energy dissipation due to internal tides, without unintentional violation of the original dynamical balances in an OGCM. Owing to this feature, the knowledge of barotropic tide modeling can be exploited to improve reproducibility of tides in an OGCM. In other words, this scheme drives an OGCM by the barotropic tidal currents which are predicted progressively by a tuned tide model, in lieu of using the equilibrium tidal potential. The numerical cost of the scheme is comparable to the barotropic calculation of the original OGCM.

We incorporated this scheme into Meteorological Research Institute Community Ocean Model (MRI.COM) and executed test experiments with a low-resolution global model $\left(1^{\circ} \times 1 / 2^{\circ}\right)$. The results showed that the model could simulate tides realistically without affecting the basic fields unintentionally, and that the model runs stably for at least one year. The root-mean-squared error of the tidal heights was only $10.0 \mathrm{~cm}$ in the reference of a data-assimilation result, suggesting that the tide reproducibility is comparable to that of tide models tuned elaborately. By contrast, when the SAL term was ignored, the reproducibility decreased significantly, as the error rose to $31.3 \mathrm{~cm}$. This suggests that the SAL scalar parameterization must be utilized in order to introduce tides into an OGCM realistically. Although this conclusion has been drawn by Arbic et al. (2010), our methodology is different from theirs.

The essential difference between the scheme of Arbic et al. (2010) and our new scheme is the approximations used to decompose the tidal and non-tidal components, as explained in Sect. 2.2. All motions in periods shorter than 25 hours are assumed to be tidal currents in the scheme of Arbic et al. (2010), while only the motions of the linear primary response to the tidal forcing are assumed to be tidal currents in our new scheme (though secondary oscillations of tides can be represented in an OGCM (Appendix)). As a result, for example, the tidal motions in the scheme of Arbic et al. (2010) include inertial oscillations at higher latitudes than $29^{\circ}$, where the inertial period is shorter than 25 hours. In our new scheme, however, it may be hard to control tides in coastal regions, 
since the nonlinearity becomes relatively strong there. By investigating how the tide reproducibility in an OGCM depends on the selection of the two schemes, validation of their approximations can be tested under realistic situations. We believe that this problem is worthy of study in order to further refine the tide schemes.

It should be noted that our model settings were rather crude. Recently, sophisticated parameterizations for tidal energy dissipation have been proposed by studies about the interaction between tides and topography (e.g. Jayne and St. Laurent, 2001). The tide reproducibility may improve further by adopting such parameterizations, making use of the feature that lets the tide settings be decided independently of which OGCM is used. Actually, our case studies showed that the reproducibility depends sharply on the configuration of the viscosity related to topography, suggesting a possible contribution of such parameterizations. Model sensitivity to bottom drag parameterizations should be investigated with this scheme, too. Though we used the Weatherly et al. (1980) and Schwiderski (1980) parameterizations for the basic and linear tidal components, respectively, they have not been tested in the situation where both of the tidal currents and the geostrophic circulations exist. Thus, implementing the appropriate tidal parameterizations in an OGCM in the framework of our new scheme remains to be carried out. In order to do this, it is also necessary to systematically evaluate errors in the phase and the amplitude of each tidal constituent, which is not analyzed in this paper. We plan to do this using our model, MRI.COM.

In spite of the crude settings, our model generally reproduced similar amplitudes of the M2 and K1 tidal currents and the tidal energy conversions when compared with previous tidal modeling studies. In addition, excitement of internal tides and enhancement of vertical mixing were found in the model, though the experimental period was as short as 40 days. We did this by generating the realistic tidal currents in the model through an explicit tidal scheme, in contrast to the indirect parameterizations of tidal mixing used by many traditional OGCMs, such as additional increase of the background vertical diffusivity. Usage of our scheme is expected to improve representation of various physical processes, such as water exchange between coastal and open oceans, and even chemical and biological processes (e.g. Sect. 8.6 of Simpson and Sharples, 2012). Explicit introduction of tides into an OGCM is a significant step towards upgrading ocean modeling. We plan to investigate the impacts in more detail using a model with a finer resolution.

\section{Appendix A}

\section{Modification of tides}

Tides affect the basic field as shown in Sect. 3.3, and in turn, the basic field modifies the tides. For example, when density stratification exists, kinetic energy conversion occurs from barotropic tides to internal tides. In the new tide scheme, the linear tidal component represents the primary barotropic response to the equilibrium tide potential, and does not include such modification. This appendix explains how such modification is represented in the framework of the new tide scheme.

To treat the question clearly and analytically, we consider a simple situation as follows. The ocean state is thoroughly horizontally uniform, including the tidal forcing, and dissipation and bottom friction are ignored. Under these assumptions, the momentum equation of the linear tidal component, Eq. (12), is simplified as

$$
\begin{aligned}
& \frac{\partial U_{l t}}{\partial t}-f V_{l t}=g H \beta \frac{\partial \eta_{0}}{\partial x} \\
& \frac{\partial V_{l t}}{\partial t}+f U_{l t}=g H \beta \frac{\partial \eta_{0}}{\partial y},
\end{aligned}
$$

where $U$ and $V$ indicate the components of the velocity vector $\boldsymbol{U}=(U, V)$. Now, introducing complex number expressions

$$
\begin{aligned}
& \boldsymbol{U}_{l t}=U_{l t}+i V_{l t} \\
& \boldsymbol{F}=\frac{\partial \eta_{0}}{\partial x}+i \frac{\partial \eta_{0}}{\partial y},
\end{aligned}
$$

Eqs. (A1) and (A2) combine to become

$$
\frac{\partial \boldsymbol{U}_{l t}}{\partial t}+\text { if } \boldsymbol{U}_{l t}=g H \beta \boldsymbol{F} .
$$

We assume a horizontal vector varying trigonometrically with frequency $\sigma$ for the tidal forcing $\boldsymbol{F}$, and as a result, both $\boldsymbol{U}_{l t}$ and $\boldsymbol{F}$ can be deformed to a sum of the two rotary components in general as follows (Davies, 1985; Sakamoto and Akitomo, 2006):

$\boldsymbol{U}_{l t}=R_{l t}^{+} e^{i \sigma t}+R_{l t}^{-} e^{-i \sigma t}$

$\boldsymbol{F}=F^{+} e^{i \sigma t}+F^{-} e^{-i \sigma t}$

where $R_{l t}^{+}$and $F^{+}$are the amplitudes of the counterclockwise components, while $R_{l t}^{-}$and $F^{-}$are those of the clockwise components. Substituting Eqs. (A6) and (A7) into Eq. (A5), we obtain the solution of $\boldsymbol{U}_{l t}$ for $\boldsymbol{F}$,

$$
\begin{aligned}
& R_{l t}^{+}=\frac{g H \beta}{i(\sigma+f)} F^{+} \\
& R_{l t}^{-}=\frac{g H \beta}{i(-\sigma+f)} F^{-} .
\end{aligned}
$$

Now that the solution for the linear tidal component is obtained, we show how the basic equations represent modification of tides induced by secondary interactions between the linear tidal component $\boldsymbol{U}_{l t}$ and the basic field. Making use of 
the assumptions of Eqs. (A1) and (A2), the barotropic momentum equation of the basic component Eq.(13), i.e., the original equation of OGCM, is simplified to

$\frac{\partial \boldsymbol{U}_{b}}{\partial t}+i f \boldsymbol{U}_{b}=\boldsymbol{X}$

where $\boldsymbol{U}_{b}$ is a complex number,

$\boldsymbol{U}_{b}=U_{b}+i V_{b}$,

and $\boldsymbol{X}$ represents the secondary interactions. Here, we assume a linear damping for $\boldsymbol{X}$. Modeling the barotropic tides as dissipated by excitation of the internal tides due to a combination of tidal currents and stratification,

$\boldsymbol{X}=-a \boldsymbol{U}_{l t}$.

The constant $a$ is a damping coefficient with units of $\mathrm{s}^{-1}$. Now we deform $\boldsymbol{U}_{b}$ to a sum of the two rotary components in the same manner as in Eq. (A6):

$\boldsymbol{U}_{b}=R_{b}^{+} e^{i \sigma t}+R_{b}^{-} e^{-i \sigma t}$.

Substituting Eqs. (A12) and (A13) into Eq. (A10), we obtain a modification of the tidal currents induced by the secondary interaction, $\boldsymbol{X}$ :

$$
\begin{aligned}
& R_{b}^{+}=\frac{-a}{i(\sigma+f)} R_{l t}^{+} \\
& R_{b}^{-}=\frac{-a}{i(-\sigma+f)} R_{l t}^{-} .
\end{aligned}
$$

The actual tidal currents are a sum of the linear tidal component, $\boldsymbol{U}_{l t}$, and the modification due to the secondary interactions. Since the secondary interaction is equal to $\boldsymbol{U}_{b}$ in the present situation, the entire tidal currents driven by the forcing $\boldsymbol{F}$ are given by Eqs. (A8), (A9), (A14) and (A15) as follows:

$$
\begin{aligned}
\boldsymbol{U}_{l t}+\boldsymbol{U}_{b} & =\left(1+\frac{-a}{i(\sigma+f)}\right) \frac{g H \beta}{i(\sigma+f)} F^{+} e^{i \sigma t} \\
& +\left(1+\frac{-a}{i(-\sigma+f)}\right) \frac{g H \beta}{i(-\sigma+f)} F^{-} e^{-i \sigma t} .
\end{aligned}
$$

This expression clearly shows how the tidal currents induced by $F^{+}$and $F^{-}$are modified by the damping $a$, which represents the secondary interaction between the tidal and basic fields. The relative magnitude of the modification in comparison to the linear tidal component is indicated by the ratio of $a$ to $\sigma+f$. This means that the modification is usually smaller than the linear tidal component, since time scales of decay of barotropic tidal currents due to excitement of internal waves (or other interaction processes) are usually larger than periods of main tidal constituents or the inertial period ( $\sim$ day). This is consistent with our test experiments, where the tides are almost entirely represented by the linear tidal component (Fig. 2).
As explained here using a simple situation, the interaction between the tidal and basic fields emerges through a driving term $(\boldsymbol{X})$ in the basic equation. Change of the basic field induced by $\boldsymbol{X}$ can be considered the modification of tides if its frequency is the same as the tidal forcing. Otherwise, the change is an excitation of other tidal constituents such as overtides, or modification of the basic field. In any case, these modification processes are represented explicitly in the framework of the original OGCM, although the governing equations of the modification currents $\boldsymbol{U}_{b}$ are different from those of the linear tidal currents $\boldsymbol{U}_{l t}$.

Acknowledgements. We thank the members of oceanographic research department of Meteorological Research Institute (MRI) for fruitful discussions and helpful comments. This work was funded by MRI and was partly supported by JSPS KAKENHI Grant Number 24740323.

Edited by: M. Hecht

\section{References}

Arbic, B. K., Garner, S. T., Hallberg, R. W., and Simmons, H. L.: The accuracy of surface elevations in forward global barotropic and baroclinic tide models, Deep-Sea Res. II, 51, 3069-3101, doi:10.1016/j.dsr2.2004.09.014, 2004.

Arbic, B. K., Wallcraft, A. J., and Metzger, E. J.: Concurrent simulation of the eddying general circulation and tides in a global ocean model, Ocean Modell., 32, 175-187, doi:10.1016/j.ocemod.2010.01.007, 2010.

Bessiéres, L., Madec, G., and Lyard, F.: Global tidal residual mean circulation: Does it affect a climate OGCM?, Geophys. Res. Lett., 35, L03 609, doi:10.1029/2007GL032644, 2008.

Davies, A. M.: On determining current profiles in oscillatory flows, Appl. Math. Modelling, 9, 419-428, doi:10.1016/0307904X(85)90107-6, 1985.

Egbert, G. D. and Ray, R. D.: Estimates of M2 tidal energy dissipation from TOPEX/Poseidon altimeter data, J. Geophys. Res., 106, 22 475-22 502, doi:10.1029/2000JC000699, 2001.

Egbert, G. D. and Ray, R. D.: Semi-diurnal and diurnal tidal dissipation from TOPEX/Poseidon altimetry, Geophys. Res. Lett., 30, 1907, doi:10.1029/2003GL017676, 2003.

Gent, P. R. and Mcwilliams, J. C.: Isopycnal Mixing in Ocean Circulation Models, J. Phys. Oceanogr., 20, 150-155, doi:10.1175/1520-0485(1990)020<0150:IMIOCM>2.0.CO;2, 1990.

Griffies, S. M. and Hallberg, R. W.: Biharmonic friction with a Smagorinsky-like viscosity for use in large-scale eddypermitting ocean models, Mon. Wea. Rev., 128, 2935-2946, doi:10.1175/1520-0493(2000)128<2935:BFWASL>2.0.CO;2, 2000.

Griffies, S. M., Biastoch, A., Böning, C., Bryan, F., Danabasoglu, G., Chassignet, E. P., England, M. H., Gerdes, R., Haak, H., Hallberg, R. W., Hazeleger, W., Jungclaus, J., Large, W. G., Madec, G., Pirani, A., Samuels, B. L., Scheinert, M., Gupta, A. S., Severijns, C. A., Simmons, H. L., Treguier, A. M., 
Winton, M., Yeager, S., and Yin, J.: Coordinated Ocean-ice Reference Experiments (COREs), Ocean Modell., 26, 1-26, doi:10.1016/j.ocemod.2008.08.007, 2009.

Hendershott, M. C.: The effects of solid earth deformation on global ocean tides, Gyophys. J. R. Astr. Soc., 29, 389-402, doi:10.1111/j.1365-246X.1972.tb06167.x, 1972.

Hunke, E. C. and Dukowicz, J. K.: An elastic-viscous-plastic model for sea ice dynamics, J. Phys. Oceanogr., 27, 1849-1867, doi:10.1175/1520-0485(1997)027<1849:AEVPMF>2.0.CO;2, 1997.

Hunke, E. C. and Dukowicz, J. K.: The elastic-viscousplastic sea ice dynamics model in general orthogonal curvilinear coordinates on a sphere: Incorporation of metric terms, Mon. Wea. Rev., 130, 1848-1865, doi:10.1175/15200493(2002)130<1848:TEVPSI>2.0.CO;2, 2002.

Jayne, S. R. and St. Laurent, L. C.: Parameterizing tidal dissipation over rough topography, Geophys. Res. Lett., 28, 811-814, doi:10.1029/2000GL012044, 2001.

Kantha, L. H. and Clayson, C. A.: Numerical Models of Oceans and Oceanic Processes, Academic Press, 2000.

Komori, N., Ohfuchi, W., Taguchi, B., Sasaki, H., and Klein, P.: Deep ocean inertia-gravity waves simulated in a high-resolution global coupled atmosphere-ocean GCM, Geophys. Res. Lett., 35, L04 610, doi:10.1029/2007GL032807, 2008.

Kurapov, A. L., Allen, J. S., and Egbert, G. D.: Combined effects of wind-driven upwelling and internal tide on the continental shelf, J. Phys. Oceanogr., 40, 737-756, doi:10.1175/2009JPO4183.1, 2010.

Large, W. G. and Yeager, S. G.: Diurnal to decadal global forcing for coean and sea-ice models: The data sets and flux climatologies, NCAR Tech. Note: TN-460+STR, CGD Division of the Natinal Center for Atmospheric Research, 2004.

Lee, H. C., Rosati, A., and Spelman, M. J.: Barotropic tidal mixing effects in a coupled climate model: Oceanic conditions in the Northern Atlantic, Ocean Modell., 11, 464-477, doi:10.1016/j.ocemod.2005.03.003, 2006.

Matsumoto, K., Takanezawa, T., and Ooe, N.: Ocean tide models developed by assimilating TOPEX/POSEIDON altimeter data into hydrodynamical model: A global model and a regional model around Japan, J. Oceanogr., 56, 567-581, doi:10.1023/A:1011157212596, 2000.

Mellor, G. L. and Kantha, L.: An ice-ocean coupled model, J. Geophys. Res., 94, 10 937-10 954, doi:10.1029/JC094iC08p10937, 1989.

Moon, J. H., Hirose, N., Yoon, J. H., and Pang, I. C.: Offshore detachment process of the low-salinity water around Changjiang Bank in the East China Sea, J. Phys. Oceanogr., 40, 1035-1053, doi:10.1175/2010JPO4167.1, 2010.

Müller, M., Haak, H., Jüngclaus, J. H., Sundermann, J., and Thomas, M.: The effect of ocean tides on a climate model simulation, Ocean Modell., 35, 304-313, doi:10.1016/j.ocemod.2010.09.001, 2010.

Munk, W. and Wunsch, C.: Abyssal recipes II: Energetics of tidal and wind mixing, Deep-Sea Res. I, 45, 1977-2010, doi:10.1016/S0967-0637(98)00070-3, 1998.

Murray, R. J.: Explicit generation of orthogonal grids for ocean models, J. Comput. Phys., 126, 251-273, doi:10.1006/jcph.1996.0136, 1996.
Nagai, T. and Hibiya, T.: Numerical simulation of tidally induced eddies in the Bungo Channel: A possible role for sporadic Kuroshio-water intrusion (kyucho), J. Oceanogr., 68, 797-806, doi:10.1007/s10872-012-0141-9, 2012.

Nakamura, T. and Awaji, T.: Tidally induced diapycnal mixing in the Kuril Straits and its role in water transformation and transport: A three-dimensional nonhydrostatic model experiment, J. Geophys. Res., 109, C09S07, doi:10.1029/2003JC001850, 2004.

Nakano, H. and Suginohara, N.: Effects of Bottom Boundary Layer parameterization on reproducing deep and bottom waters in a world ocean model, J. Phys. Oceanogr., 32, 1209-1227, 2002.

Niwa, Y. and Hibiya, T.: Estimation of baroclinic tide energy available for deep ocean mixing based on three-dimensional global numerical simulations, J. Oceanogr., 67, 493-502, doi:10.1007/s10872-011-0052-1, 2011.

Osafune, S. and Yasuda, I.: Bidecadal variability in the intermediate waters of the northwestern subarctic Pacific and the Okhotsk Sea in relation to 18.6-year period nodal tidal cycle, J. Geophys. Res., 111, C05 007, doi:10.1029/2005JC003277, 2006.

Osborne, J. J., Kurapov, A. L., Egbert, G. D., and Kosro, P. M.: Spatial and temporal variability of the M2 internal tide generation and propagation on the Oregon Shelf, J. Phys. Oceanogr., 41, 2037-2062, doi:10.1175/JPO-D-11-02.1, 2011.

Pereira, F. P., Beckmann, A., and Hellmer, H. H.: Tidal mixing in the southern Weddell Sea: Results from a three-dimensional model, J. Phys. Oceanogr., 32, 2151-2170, doi:10.1175/15200485(2002)032<2151:TMITSW>2.0.CO;2, 2002.

Polzin, K. L.: Mesoscale eddy-internal wave coupling. Part I: Symmetry, wave capture, and results from the Mid-Ocean Dynamics Experiment, J. Phys. Oceanogr., 38, 2556-2574, doi:10.1175/2008JPO3666.1, 2008.

Postlethwaite, C. F., Maqueda, M. A. M., Fouest, V. L., Tattersall, G. R., Holt, J., and Willmott, A. J.: The effect of tides on dense water formation in Arctic shelf seas, Ocean Sci., 7, 203-217, doi:10.5194/os-7-203-2011, 2011.

Prather, M. J.: Numerical advection by conservation of second-order moments, J. Geophys. Res., 91, 6671-6681, doi:10.1029/JD091iD06p06671, 1986.

Robertson, R.: Internal tides and baroclinicity in the southern Weddell Sea 1. Model description, J. Geophys. Res., 106, 27 00127 016, doi:10.1029/2000JC000475, 2001a.

Robertson, R.: Internal tides and baroclinicity in the southern Weddell Sea 2. Effects of the critical latitude and stratification, J. Geophys. Res., 106, 27 017-27 034, doi:10.1029/2000JC000476, $2001 b$.

Sakamoto, K. and Akitomo, K.: Instabilities of the tidally induced bottom boundary layer in the rotating frame and their mixing effect, Dyn. Atmos. Oceans, 41, 191-211, doi:10.1016/j.dynatmoce.2006.06.001, 2006.

Sakamoto, K. and Akitomo, K.: The tidally induced bottom boundary layer in the rotating frame: Similarity of turbulence, J. Fluid Mech., 615, 1-25, doi:10.1017/S0022112008003340, 2008.

Sakamoto, K. and Akitomo, K.: The tidally induced bottom boundary layer in the rotating frame: Development of the turbulent mixed layer under stratification, J. Fluid Mech., 619, 235-259, doi:10.1017/S0022112008004503, 2009.

Schiller, A.: Effects of explicit tidal forcing in an OGCM on the water-mass structure and circulation in the Indonesian through- 
flow region, Ocean Modell., 6, 31-49, doi:10.1016/S14635003(02)00057-4, 2004.

Schiller, A. and Fiedler, R.: Explicit tidal forcing in an ocean general circulation model, Geophys. Res. Lett., 34, L03 611, doi:10.1029/2006GL028363, 2007.

Schwiderski, E. W.: On charting global ocean tides, Rev. Geophys. Space Phys., 18, 243-268, doi:10.1029/RG018i001p00243, 1980.

Simmons, H. L., Hallberg, R. W., and Arbic, B. K.: Internal wave generation in a global baroclinic tide model, Deep-Sea Res. II, 51, 3043-3068, doi:10.1016/j.dsr2.2004.09.015, 2004.

Simpson, J. H. and Hunter, J. R.: Fronts in the Irish Sea, Nature, 250, 404-406, doi:10.1038/250404a0, 1974.

Simpson, J. H. and Sharples, J.: Introduction to the physical and biological oceanography of shelf seas, Cambridge University Press, 2012.

St. Laurent, L. and Garrett, G.: The role of internal tides in mixing the deep ocean, J. Phys. Oceanogr., 32, 2882-2899, doi:10.1175/1520-0485(2002)032<2882:TROITI>2.0.CO;2, 2002.

Taylor, G. I.: Tidal friction in the Irish Sea, Phil. Trans. R. Soc. Lond. A, 220, 1-33, doi:10.1098/rsta.1920.0001, 1920.

Thomas, M., Jürgen Sündermann, and Maier-Reimer, E.: Consideration of ocean tides in an OGCM and impacts on subseasonal to decadal polar motion excitation, Geophys. Res. Lett., 28, $2457-$ 2460, doi:10.1029/2000GL012234, 2001.
Tsujino, H., Motoi, T., Ishikawa, I., Hirabara, M., Nakano, H., Yamanaka, G., Yasuda, T., and Ishizaki, H.: Reference manual for the Meteorological Research Institute Community Ocean Model (MRI.COM) version 3, Technical reports of the Meteorological Research Institute 59, Meteorological Research Institute, Japan, 2010.

Tsujino, H., Hirabara, M., Nakano, H., Yasuda, T., Motoi, T., and Yamanaka, G.: Simulating present climate of the global oceanice system using the Meteorological Research Institute Community Ocean Model (MRI.COM): simulation characteristics and variability in the Pacific sector, J. Oceanogr., 67, 449-479, doi:10.1007/s10872-011-0050-3, 2011.

Umlauf, L. and Burchard, H.: A generic length-scale equation for geophysical turbulence models, J. Marine Res., 61, 235-265, doi:10.1357/002224003322005087, 2003.

Weatherly, G. L., Blumsack, S. L., and Bird, A. A.: On the effect of diurnal tidal currents in determining the thickness of the turbulent Ekman bottom boundary layer, J. Phys. Oceanogr., 10, 297-300, doi:10.1175/1520-0485(1980)010<0297:OTEODT>2.0.CO;2, 1980.

Xing, J. and Davies, A. M.: The influence of wind effects upon internal tides in shelf edge regions, $J$. Phys. Oceanogr., 27, 2100-2125, doi:10.1175/15200485(1997)027<2100:TIOWEU>2.0.CO;2, 1997. 